\title{
THE HYDROPHILOUS FLORA AND VEGETATION \\ OF THE TIMIS DRAINAGE BASIN (BANAT, ROMANIA)
}

\author{
Constantin DR $\breve{A G U L E S C U} *$ \\ * "Lucian Blaga" University of Sibiu, Faculty of Sciences, Department of Ecology and \\ Environment Protection, Dr. Raţiu Street 5-7, Sibiu, Sibiu County, Romania, RO-550012, \\ ctindrg@yahoo.com
}

DOI: 10.2478/trser-2013-0034

KEYWORDS: Romania, Banat, Timiş Valley, hydrophilous, hygrophilous cormoflora, vegetation, chorology.

\section{ABSTRACT}

The paper lists 285 plant species, and 63 aquatic and paludal phytocoenoses, noted across the Timiş drainage basin during the past two centuries (the sign "!" indicates that the author saw such plants or phytocoenoses in the mentioned place).

For each individual species, I noted the scientific denomination, the author, the corresponding family, the bioform, the floristic element, ecological indices for humidity $(\mathrm{H})$, temperature $(\mathrm{T})$, and soil reaction $(\mathrm{R})$, coenotic preference and chorology in the Timiş drainage basin.

ZUSAMMENFASSUNG: Die hydrophile und hygrophile Flora und Vegetation im Einzugsgebiet des Timiş Flusses.

Es werden 285 Pflanzenarten sowie 63 Wasser- und Sumpfpflanzengesellschaften aufgezählt, die im Einzugsgebiet des Timiş-Flusses im Verlauf von zwei Jahrhunderten festgestellt wurden (das hinzugefügte Ausrufezeichen weist darauf hin, dass der Autor die jeweilige Pflanze bzw. Pflanzengesellschaft selbst an dem angegebenen Fundort vorgefunden hat).

Für jede Art ist ihr wissenschaftlicher Namen, Verfasser, Zugehörigkeit zur jeweiligen Pflanzenfamilie, Lebensform und Florenelement angegeben; hinzu kommen die ökologischen Zeigerwerte für Feuchtigkeit (U), Temperatur (T) und Bodenreaktion (R), zönotische Präferenzen sowie die Chorologie der Arten im Einzugsgebiet des TimişFlusses.

REZUMAT: Flora şi vegetaţia hidrofilă a bazinului de drenaj Timiş.

În lucrare, sunt enumerate 285 specii de plante şi 63 asociaţii vegetale acvatice şi palustre, semnalate în bazinul Văii Timişului pe parcursul a două secole (semnul ! evidenţiază faptul că autorul a văzut plantele sau fitocenozele asociaţiilor în locul citat).

La fiecare specie vegetală s-a notat denumirea ştiinţifică, autorul, familia la care aparţine, bioforma, elementul floristic, indicii ecologici pentru umiditate $(U)$, temperatură $(T)$, şi reacţia solului (R), de asemenea preferinţele cenotice şi corologia în bazinul Timişului. 


\section{INTRODUCTION}

The first information regarding the flora of Banat province (including aquatic and paludal plants) comes from the 19th-century botanists Rochel (1823), Heuffel (1858) and Borbás (1884). More than half a century later, several botanical works are published by Buia (1942), Soran (1954, 1956) and particularly Boşcaiu (1942, 1944, 1965, 1966, 1971, 1971a), in which the authors list over two hundred species of hydrophilic, hygrophilous, and mezohygrophile plant species, as well as thirty conenoses in such ecological categories. Stere (1971, 1971a) notes species and conenoses in the Timiş-Bega rivers area; Vicol (1974) in the Lugoj Piedmont; Arvat (1977) in and among the rivers Timiş, Pogoniş and Bârzava; Pop (1977) in the lower Timiş River plain. Recently, Neacşu et al. $(2008,2009)$ have added data regarding aquatic and paludal species with information from Liebling. Finally, the undersigned researched the flora and vegetation of the Timiş from Trei Ape up to Giulvăz (in 2001, 2008 and 2011).

\section{MATERIAL AND METHODS}

The paper was developed based on field observation made by the author and information taken from the bibliography to be found at the end of the paper. While in the field, I noted plant species and phytocoenoses (in most cases, including the execution of phytocoenologic samplings). For each individual species, I noted the scientific denomination, the author, the corresponding family, the bioform, the floristic element, ecological indices for humidity $(\mathrm{H})$, temperature $(\mathrm{T})$, and soil reaction $(\mathrm{R})$, coenotic preference and chorology in the Timiş drainage basin.

\section{RESULTS AND DISCUSSIONS}

The paper lists 285 plant species and 63 aquatic and paludal phytocoenoses observed across the Timiş drainage basin (the sign "!" indicates that the author saw such plants or phytocoenoses in the place mentioned). For each individual species, I noted the scientific denomination, the author, the corresponding family, the bioform, the floristic element, ecological indices for humidity $(\mathrm{H})$, temperature $(\mathrm{T})$, and soil reaction $(\mathrm{R})$, coenotic preference and chorology in the Timiş drainage basin.

\section{Flora}

\section{Equisetaceae}

Equisetum fluviatile L. (E. limosum L.): Hh, Cp; U5T3R0, Magnocaricion elatae, Phragmitetea: Bistra Mărului Valley (Boşcaiu, 1971)

Equisetum hyemale L.: G, Cp; U3.5T2.5R4, Alno-Padion:, Armeniş on Alb River and Lung River, Borlova, Hidegu Stream, Poiana Mărului, Rusca, (Boşcaiu, 1971)

Equisetum palustre L.: G, Cp; U5T2R0, Molinietalia: Belinţ, Caransebeş (!), Glimboca, Lugoj (!), Măgura (Boşcaiu, 1971), Marga (!), Obreja, Hidegu Stream, Rusca (Boşcaiu, 1971), Trei Ape (!), Turnu Ruieni, Bistra Valley, Zerveşti (Boşcaiu, 1971)

Equisetum sylvaticum L.: G, Cp; U3.5T2R0, Alno-Padion, Alnion glutinosae-incanae: Borlova, V. Bistra Mărului (Boşcaiu, 1971), Trei Ape (!)

Equisetum telmateia Ehrh. (E. maximum Lam.): G, Cp; U3.5T2R0, Alno-Padion, Eriophorion latifolii, Filipendulo-Petasition: Armeniş on Alb River, Borlova, Sebeşului Spring, Hidegu Stream, Rusca, Teregova, Bistra Mărului Valley, (Boşcaiu, 1971), Armeniş (!), Trei Ape (!); f. comosum (Milde) Aschers.: Armeniş (Flora I) 


\title{
Ophioglossaceae
}

Ophioglossum vulgatum L.: G, Cp; U4T3R0, Molinion coeruleae: Bazoş, Zerveşti (Flora I)

\author{
Athyriaceae \\ Matteuccia struthiopteris (L.) Todaro (Struthiopteris filicastrum All.): H, Cp; U4T2R0, Alno- \\ Padion: Trei Ape (!)
}

\section{Thelypteridaceae}

Thelypteris palustris Schott (Dryopteris thelypteris (L.) A. Gray): Hh-G, Cp; U4T0R3, Alnion glutinosae, Alno-Padion, Magnocaricion elatae: Obreja (Boşcaiu, 1971)

\section{Marsileaceae}

Marsilea quadrifolia L.: Hh, Eua (M); U6T3R0, Nanocyperion: Liebling, Moşniţa (Soran, 1954), Urseni (Stere, 1971a)

\section{Salviniaceae}

Salvinia natans (L.) All.: Hh, Eua; U6T3R3, Hydrocharition: Albina, Bazoş, Chizătău, Cruceni, Giulvăz, Ivanda (Stere, 1971a), Liebling in fen Tofaia (Soran V., 1956), Lugoj in the lake from Str. Bocşei, at the brick yard Bartoş and in lakes from Complexul III (Boşcaiu, 1966), Pădureni on Timişul Mort (Soran, 1956), Şag, Uliuc, Urseni (Stere, 1971a), Unip on Timiş (Flora I)

\section{Nymphaeaceae}

Nuphar lutea (L.) Sm.: Hh, Eua-M; U6T0R3.5, Nymphaeion: between Lugoj and Tapia on Ştiuca Stream (Boşcaiu, 1966), Ciacova, Lugoj, Timiş tributaries (Flora III)

Nymphaea alba L.: Hh, E; U6T0R4, Nymphaeion: Lugoj (Flora III)

\section{Ceratophyllaceae}

Ceratophyllum demersum L. subsp. demersum: Hh, Cosm; U6T3R0, Potamion: Lugoj (Flora III)

Ceratophyllum submersum L.: Hh, E; U6T3.5R0, Hydrocharition, Potamion: Caransebeş (!), Chizătău, Cruceni, Diniaş, Foeni, Giroc (Stere, 1971a), Lugoj (!) in the lake from Str. Bocşei, at the brick yard Bartoş (Boşcaiu, 1966), between Lugoj and Tapia on Ştiuca Stream (Boşcaiu, 1966), Moşniţa Veche, Rudna (Stere, 1971a), Pădureni on Timişul Mort and in puddles (Soran, 1956)

\section{Ranunculaceae}

Caltha palustris L.: H, Cp; U4.5T0R0, Calthion palustris, Cardamini-Montion, Molinietalia: Lugoj (Boşcaiu, 1966), Mt. Mic (Boşcaiu, 1971), Trei Ape (!); var. alpina (Schur) Graebn.: Semenic Mts. (Flora II), Armeniş on Alb River and Lung River, Borlova, Sebeşului Spring, Marga, Măgura, Hidegu Stream, Poiana Mărului, Poiana Nedeii, Rusca, Turnu Ruieni, Bistra Mărului Valley (Boşcaiu, 1971); var. alpestris (Schott, Nym. et Korschy) Beck: Bistra Mărului Spring, Mic Mt. (Boşcaiu, 1971, 1971a), Căleanu Peak (Boşcaiu, 1971), Ţarcu Peak (Boşcaiu, 1971, 1971a), Sebeşului Valley (Boşcaiu, 1971a)

Myosurus minimus L.: Th, Cosm; U4T4R3, Agropyro-Rumicion, Nanocyperion flavescentis: Ciacova (Flora II) 
Ranunculus aquatilis L.: Hh, Cosm; U6T4R0, Potamion, Nymphaeion: Dragşna, Foeni (Stere, 1971a), Liebling (Neacşu et al., 2008)

Ranunculus flammula L.: H, Eua; U4.5T3R0, Agrostion stoloniferae, Caricion canescentinigrae, Magnocaricion elatae: Caransebeş-Gara Ţiglărie (Boşcaiu, 1965), Bistra Valley beside Măgura (Boşcaiu, 1971)

Ranunculus lateriflorus DC.: Th, Eua(C); U5T3R5, Nanocyperion, Beckmannion: Diniaş, Giulvăz (Stere, 1971a)

Ranunculus lingua L.: Hh, Eua; U6T3R4, Phragmition australis: Lugoj at the brick yard Bartoş (Boşcaiu, 1966)

Ranunculus ophioglossifolius Vill.: H, Atl-M; U5T3,5R0, Calthion: Peciu Nou (Flora XIII) Ranunculus repens L.: H, Eua; U4T0R0, Agropyro-Rumicion, Alno-Padion, Bidentetalia tripartiti, Calystegion, Molinio-Arrhenatheretea, Phragmitetea, Plantaginetea majoris, Salicetea purpureae: Armeniş (!) on Alb River and Lung River (Boşcaiu, 1971), Bazoş, Belinţ (!), Bolvaşniţa (Boşcaiu, 1971, !), Borlova, Caransebeş, Chizătău, Coşteiu, Criciova, Gărâna, Giulvăz (!), Glimboca (Boşcaiu, 1971, !), Ilova, Sebeşului Spring (Boşcaiu, 1971), Liebling (Neacşu et al., 2008), Lugoj (Boşcaiu, 1966, !), Lugojel (!), Marga, Măgura (Boşcaiu, 1971,!), Ţarcu Peak, Obreja (Boşcaiu, 1971), Oţelul Roşu (Boşcaiu, 1971, !), Peciu Nou (!), Hidegu Stream, Poiana Mărului, Rusca, Sadova Nouă, Sadova Veche (Boşcaiu, 1971), Sacu, Şag, Trei Ape (!), Bistra Mărului Valley, Sebeşului Valley (Boşcaiu, 1971, 1971a), Vălişoara, Vârciorova, Zăvoi, Zlagna (Boşcaiu, 1971), between Zerveşti and Turnu Rueni (Boşcaiu, 1965, 1971); f. haynaldi (Menyh) Borza.: Giroc (Buia, 1942)

Ranunculus sardous Cr.: Th-TH, Eua; U3T3R4, Agropyro-Rumicion, Agrostion stoloniferae, Nanocyperion: Jebel, Liebling (Soran, 1956)

Ranunculus trichophyllus Chaix. (Batrachium trichophyllum (Chaix) Bosch, B. divaricatum (Schrank) Wimmer): Hh, E; U6T3R0, Potamion: Caransebeş (Flora II), Liebling in Balta Mare and Balta Mică (Soran, 1956)

Thalictrum flavum: H, Eua; U4,5T0R4,5, Molinietalia, Filipendulo-Petasition, Alno-Padion: Caransebeş-Gara Ţiglărie (Boşcaiu, 1965), Liebling (Neacşu et al., 2008), between Zerveşti and Turnu Rueni (Boşcaiu, 1965)

Thalictrum lucidum L.: H, Ec; U4.5T3R5, Alnetea glutinosae, Alno-Padion, FilipenduloPetasition, Molinietalia, Salicetea purpureae: Hidegu Stream, Poiana Mărului (Boşcaiu, 1971)

Thalictrum simplex L. subsp. galioides (Nestl.) Borza: H, Eua; U4T3R0, Molinion: Caransebeş-Gara Ţiglărie (Boşcaiu, 1965), Teregova (Boşcaiu, 1971), between Zerveşti and Turnu Rueni (Boşcaiu, 1965, 1971)

\section{Betulaceae}

Alnus glutinosa (L.) Gaerter: Mph-mPh, Eua; U5T3R3, Alnion glutinosae, Alno-Padion: upstream of Armeniş (!) on Alb River and Lung River, Borlova (Boşcaiu, 1971), Buchin, Bucoşniţa, Gărâna (!), Măgura, Obreja, Hidegu Stream (Boşcaiu, 1971), Petroşniţa (!), Poiana Mărului, Rusca, Sadova Veche (Boşcaiu, 1971, !), Teregova, Trei Ape (!), Turnu Ruieni, Bistra Valley, Bistra Mărului Valley, Bolvaşniţa Valley, Sebeşului Valley, Var, Vălişoara, Vârciorova, Zervești (Boșcaiu, 1971)

Alnus incana (L.) Moench: Mph-mPh, E; U4T2R4, Alno-Padion, Salicion albae: Armeniş (!) on Alb and Lung River, Borlova, Bucova, Ilova, Sebeşului Spring, Hidegu Stream, Poiana Mărului, Poiana Nedeii, Rusca, Sadova Nouă, Turnu Ruieni, Bistra Valley, Bistra Mărului Valley, Var, Vălişoara, Vârciorova, Zerveşti, Zlagna (Boşcaiu, 1971) 
Alnus $x$ pubescens Tausch. (glutinosa x incana): Armeniş, Poiana Mărului, Bistra Valley at Măgura, Bistra Mărului Valley (Boşcaiu, 1971)

\section{Portulacaceae}

Montia minor C. C. Gmelin (M. verna Necker, M. fontana L.): Th, Cp; U4.5T3.5R2.5, Alnetalia glutinosae, Cardamini-Montion, Nanocyperion flavescentis: Belinţ (Stere, 1971), Caransebeş, Lugoj (Bujorean, 1959), Sacu (Stere, 1971, Flora XIII); subsp. chondrosperma Cham.: Sacu (Herb. Grăd. Bot. Iaşi)

\section{Caryophyllaceae}

Lychnis flos-cuculi L.: H, Eua; U4T2.5R0, Magnocaricion elatae, Molinietalia, MolinioArrhenatheretea: Armeniş (!) on Alb River, Borlova (Boşcaiu, 1971), Caransebeş (!), Caransebeş-Gara Țiglărie (Boşcaiu, 1965), Glimboca, Sebeşului Spring (Boşcaiu, 1971), Mic Mt. (Boşcaiu, 1971a), Obreja, Hidegu Stream, Poiana Mărului, Bistra Valley, Bistra Mărului Valley (Boşcaiu, 1971), between Zerveşti and Turnu Rueni (Boşcaiu, 1965)

Myosoton aquaticum (L.) Moench (Stellaria aquatica (L.) Scop.): H, Eua; U4T3R0, AlnoPadion, Bidention tripartiti, Salicion albae, Senecion fluviatilis: Armeniş (!) on Alb River, Borlova (Boşcaiu, 1971), Caransebeş (!), Hidegu Stream, Măgura, Poiana Mărului, Rusca (Boşcaiu, 1971), Trei Ape (!), Turnu Ruieni, Bistra Valley, Bistra Mărului Valley, Zerveşti (Boşcaiu, 1971)

Stellaria uliginosa Murray (S. alsine Grimm.): H, Cp; U4.5T2.5R2.5, Cardamini-Montion: Armeniş (!), Trei Ape (!)

\section{Polygonaceae}

Polygonum amphibium L.: Hh, Cosm; U6T3R0, Agropyro-Rumicion, Agrostion stoloniferae, Alnetea glutinosae, Phragmitetea, Polygono-Chenopodion polyspermi, Salicetea purpureae: Chizătău (!), Liebling (Neacşu et al., 2008); f. aquaticum Leyss: Boldur, Lugoj at the brick yard Bartoş (Boşcaiu, 1966)

Polygonum bistorta L.: G, Eua; U4T2.5R3, Calthion palustris, Molinietalia, TrisetoPolygonion: Semenic Mt. (Flora I), Mic Mt. (Boşcaiu, 1971, 1971a)

Polygonum cuspidatum Sieb. et Zucc. (Reynoutria japonica Houtt.): G, Adv; U3.5T3R4, Alno-Padion, Calystegion, Salicion albae: Armeniş (!), Bucoşniţa (!), Obreja (Boşcaiu, 1971), Petroşniţa (!), Sadova Veche (!), Bistra Valley between Glimboca and Măgura (Boşcaiu, 1971) Polygonum hydropiper L.: Th, Cp; U4.5T3R4, Alnetea glutinosae, Bidention tripartiti, Salicion albae: Armeniş (Boşcaiu, 1971, !), Borlova (Boşcaiu, 1971), Boldur (Boşcaiu, 1966), Buchin, Bucoşniţa, Caransebeş, Giulvăz (!), Glimboca (Boşcaiu, 1971, !), Liebling (Neacşu et al., 2008), Lugoj (Boşcaiu, 1966, !), Măgura, Obreja (Boşcaiu, 1971), Oţelul Roşu, Peciu Nou (!), Hidegu Stream, Rusca, Sadova Veche, Turnu Ruieni, Bistra Mărului Valley, Var, Vălişoara, Vârciorova, Zerveşti (Boşcaiu, 1971)

Polygonum lapathifolium L. ssp. lapathifolium: Th, Cosm; U4T0R3, Bidention tripartiti, Polygono-Chenopodion polyspermi, Sisymbrion officinalis: Armeniş (!), Borlova (Boşcaiu, 1971), Caransebeş, Glimboca (!), Lugoj (Boşcaiu, 1966, !), Lugojel (!), Măgura, Obreja, Rusca, Sadova Veche, Turnu Ruieni, Bistra Valley, Var, Zerveşti (Boşcaiu, 1971)

Polygonum minus Hudson: Th, Eua; U4,5T3R4, Bidention: Obreja, Bistra Valley beside Măgura, Bistra Mărului Valley (Boşcaiu, 1971)

Polygonum mite Schrank: Th, E; U5T3R4, Bidentetalia tripartiti: Liebling (Neacşu et al., 2008), Lugoj (Boşcaiu, 1966, !), Obreja, Bistra Valley beside Măgura, Bistra Mărului Valley (Boşcaiu, 1971) 
Polygonum persicaria L.: Th, Eua; U4.5T3R0, Phragmitetea, Polygono-Chenopodietalia, Salicetalia purpureae: Armeniş (!), Jebel, Liebling (Soran, 1956), Teregova (!)

Rumex conglomeratus Murray: $\mathrm{H}, \mathrm{Cp}$; U4T3R4, Agropyro-Rumicion, Bidention tripartiti: Armeniş (Boşcaiu, 1971,!), Bucoşniţa, Caransebeș (!), Lugoj (Flora I,!), Obreja, Rusca, Turnu Ruieni, Bistra Valley, Bistra Mărului Valley, Zerveşti (Boşcaiu, 1971),

Rumex crispus L.: H, Eua; U4T3R0, Agropyro-Rumicion, Arrhenatherion elatioris: Armeniş, Borlova (Boşcaiu, 1971, !), Bucoşniţa, Caransebeş (!), Glimboca, Măgura (Boşcaiu, 1971, !), Jebel (Soran, 1956), Liebling (Neacşu et al., 2008, Soran, 1956), Hidegu Stream, Poiana Mărului, Rusca (Boşcaiu, 1971), Trei Ape (!), Turnu Ruieni, Bistra Mărului Valley, Zerveşti (Boşcaiu, 1971)

Rumex hydrolapathum Hudson: Hh, E; U6T4R4, Phragmition australis: V. Ştiuca beside Tapia (Boşcaiu, 1966)

Rumex palustris Sm (R. limosus Thiull.): Th-TH, Eua; U5T3R4, Bidention: Liebling (Neacşu et al., 2008)

Rumex sanguineus L.: H, E; U4T3R4, Alno-Padion, Fagetalia silvaticae: Armeniș (!) on Alb and Lung River, Borlova, Sebeşului Sping, Măgura, Poiana Mărului, Rusca, Bistra Mărului Valley (Boşcaiu, 1971)

Rumex $x$ gayeri Rech f. (crispus x kerneri): Lugoj (Flora I)

\section{Saxifragaceae}

Chrysosplenium alternifolium L.: H, Cp; U4T2R4, Alno-Padion, Fagetalia silvaticae: Mt. Semenic (Flora IV), Armeniş, Borlova, Bucova, Izvorul Sebeşului, Marga, Măgura, Oțelul Roşu, Pâr. Hidegu, Poiana Mărului, Rusca, V. Bistra, Vf. Călianu, Vf. Piga, Zăvoi (Boşcaiu N., 1971), Groapa Bistrei, Mt. Mic, Mt.. Ţarcu, V. Bistra Mărului, V. Sebeşului (Boşcaiu N., 1971, 1971a)

Saxifraga aizoides L.: Ch, Eua; U4,5T0R4,5, Cratoneurion commutati: Dunga Gropii Bistra under Vf. Ţarcu (Boşcaiu N., 1971a)

Saxifraga stellaris L. subsp. robusta (Engler) Gremli: Ch, Eua (arct-alp); U5T1.5R3, Cardamini-Montion: Groapa Bistrei, Mt. Mic (Boşcaiu N., 1971, 1971a), Mt. Țarcu (Flora IV, Boşcaiu N., 1971, 1971a), V. Bistra Mărului (Boşcaiu N., 1971, 1971a), Vf. Călianu, Vf. Piga (Boşcaiu N., 1971)

\section{Parnassiaceae}

Parnassia palustris L.: H, Cp; U4.5T2R4.5, Caricetalia davallianae, Molinion coeruleae, Tofieldietalia: Groapa Bistrei (Boşcaiu N., 1971, 1971a), Mt. Mic, Mt. Ţarcu (Boşcaiu N., 1971, 1971a), Vf. Căleanu, (Boşcaiu N., 1971)

\footnotetext{
Rosaceae

Filipendula ulmaria (L.) Maxim.: H, Eua; U4.5T2R0, Alno-Padion, Filipendulo-Petasition, Molinietalia; Caransebeş, Gărâna (!), Măgura, Obreja, Pâr. Hidegu, Poiana Mărului, Rusca (Boşcaiu N., 1971), Trei Ape (!), V. Bistra, V. Bistra Mărului, Zerveşti (Boşcaiu N., 1971)

Geum rivale L.: H, Cp; U4.5T0R4.5, Adenostylion alliariae, Calthion palustris, FilipenduloPetasition: Mt. Mic (Boşcaiu N., 1971a), Mt. Ţarcu (Flora IV, Boşcaiu N., 1971), Mt. Semenic (Flora IV)

Potentilla anserina L.: H, Cosm; U4T3R4, Bidentetalia tripartiti, Molinietalia, Nanocyperetalia, Plantaginetalia majoris: Belinț (!), Borlova (Boşcaiu N., 1971), Chizătău, Coşteiu, Giulvăz (!), Liebling (Neacşu A. et al., 2008), Lugoj, Lugojel, Peciu Nou, Sacu, Şag (!), V Bistra, Vârciorova, (Boşcaiu N., 1971)
} 
Potentilla supina L.: TH-H, Eua-sM; U4T3R0, Bidention tripartiti, Nanocyperion flavescentis: V. Bistra (Boşcaiu N., 1971)

Sanguisorba officinalis L.: H, Cp; U3T3R0, Molinietalia: Caransebeş-Gara Ţiglărie (Boşcaiu N., 1965; Flora IV), Moşniţa (Flora IV), V. Bistra, (Boşcaiu N., 1971), between Zerveşti and Turnu Rueni (Boşcaiu N., 1965, 1971)

\section{Fabaceae}

Lotus pedunculatus Cav. (Lotus uliginosus Schkuhr): H, Atl-sM; U4T3R3, Molinietalia: Lugoj (Boşcaiu N., 1966)

Trifolium hybridum L. ssp. hybridum: H, Atl-E; U3.5T3R4, Agropyro-Rumicion, Agrostion stoloniferae, Calthion palustris: Armeniş (Boşcaiu N., 1971,!), Borlova (Boşcaiu N., 1971), Caransebeş (!), Caransebeş-Gara Ţiglărie (Boşcaiu N., 1965), Lugoj (Boşcaiu N., 1966), Marga, Obreja, Pâr. Hidegu, Poiana Mărului, Rusca (Boşcaiu N., 1971), V. Bistra (Boşcaiu N., 1971), between Zerveşti and Turnu Rueni (Boşcaiu N., 1965, 1971); subsp. elegans (Savi) Acherson et Graebner: Sadova Veche (Boşcaiu N., 1971 ap. V. Borbas, 1873)

\section{Haloragaceae}

Myriophyllum spicatum L.: Hh, Cp; U6T0R4.5, Potamion, Nymphaeion: Liebling in Balta Mare and Balta Mică (Soran V., 1956), Lugoj (!) in the lake from Str. Bocşei and in lakes from Complexul III (Boşcaiu N., 1966), between Şag and Urseni in Timiş (Soran V., 1956), Urseni (Stere G., 1971a)

Myriophyllum verticillatum L.:Hh, Cp; U6T3,5R3,5, Potamion, Nymphaeion: Rudna (Stere G., 1971a)

\section{Lythraceae}

Lythrum hyssopifolia L.: Th, Cosm; U4T3R0, Nanocyperion flavescentis: Armeniş (Boşcaiu N., 1971), Jebel (Soran V., 1956), Liebling (Soran V., 1954, 1956), Lugoj in the lake from Str. Bocşei (Boşcaiu N., 1966), Obreja (Boşcaiu N., 1971)

Lythrum portula (L.) D.A. Webb. (Peplis portula L.): Th, Atl-M; U4T3R0, Nanocyperion flavescentis: Bucoşniţa (!), Glimboca (Boşcaiu N., 1971), Jebel, Liebling (Soran V., 1954, 1956), Lugoj (Boşcaiu N., 1966, !), Pâr. Hidegu, Obreja (Boşcaiu N., 1971), Trei Ape (!),

Lythrum salicaria L.: H, Cp; U4T3R0, Alnetea glutinosae, Filipendulo-Petasition, Molinietalia, Phragmitetea, Salicetea purpureae: Armeniş (Boşcaiu N., 1971,!), Belinţ (!), Borlova (Boşcaiu N., 1971), Bucoşniţa, Caransebeş, Gărâna, Giulvăz (!), Glimboca, Ilova, Izvorul Sebeşului (Boşcaiu N., 1971), Liebling (Neacşu A. et al., 2008), Lugoj (Boşcaiu N., 1966,!), Marga, Măgura, Obreja, Oţelul Roşu, Pâr. Hidegu (Boşcaiu N., 1971), Pădureni on Timişul Mort (Soran V., 1956), Poiana Mărului, Rusca (Boşcaiu N., 1971), Sadova Veche (Boşcaiu N., 1971,!), Tapia (Boşcaiu N., 1966), Teregova (!), Turnu Ruieni, V. Bistra, Var, Zerveşti (Boşcaiu N., 1971)

Lythrum virgatum L.: H, Eua-C; U4,5T3,5R4, Agrostion stoloniferae: Jebel, Liebling (Soran V., 1956)

\section{Onagraceae}

Chamerion (Chamaenerion) dodonei (Vill.) Holub (Chamaenerion palustre auct., Epilobium dodonaei Vill.): H, Ec; U4.5T0R2, Thlaspietea rotundifolii: Măguri, Sacu in V. Timişului (Flora V) 
Epilobium alsinifolium Vill.: H, E (arct-alp); U5T1.5R0, Montio-Cardaminetea: Groapa Bistrei (Boşcaiu N., 1971), Mt. Ţarcu (Flora V, Boşcaiu N., 1971), Poiana Mărului (Boşcaiu N., 1971)

Epilobium anagalidifolium Lam. (E. alpinum auct. non L.): H, Cp (arct-alp); U4T1.5R0, Androsacetalia alpinae, Cardamini-Montion: Groapa Bistrei, Mt. Ţarcu (Flora V, Boşcaiu N., 1971), Izvorul Hidegu (Boşcaiu N., 1971)

Epilobium ciliatum Rafin (E. adenocaulon Hausskn, E. hirsutum var. adenocaulon Hausskn.).: H, Adv; U4T3R3, Filipendulo-Petasition, Molinietalia, Phragmitetea

Epilobium hirsutum L.: H, Eua; U4T3R3, Filipendulo-Petasition, Phragmitetea: Armeniş (Boşcaiu N., 1971,!), Borlova (Boşcaiu N., 1971), Bucoşniţa, Caransebeş (!), Glimboca (Boşcaiu N., 1971), Lugoj (Boşcaiu N., 1966,!), Pădureni on Timişul Mort (Soran V., 1956), Pâr. Hidegu, Poiana Mărului, Rusca (Boșcaiu N., 1971), Sadova Veche (!), Teregova (!), Trei Ape (!),V. Bistra (Boşcaiu N., 1971); var. villosum (Roch.) Hausskn.: Borlova (Boşcaiu N., 1971)

Epilobium nutans F.W. Schmidt: H, E (alp); U5T2R2, Cardamini-Montion, Sphagnion fusci: Groapa Bistrei (Flora V), Mt. Mic (Boşcaiu N., 1971, 1971a), Mt. Ţarcu (Flora V, Boşcaiu N., 1971, 1971a)

Epilobium obscurum Schreber: H, E; U5T0R2, Cardamini-Montion, Epilobietea angustifolii, Glycerio-Sparganion: Caransebeş (!)

Epilobium palustre L.: H, Cp; U5T0R2, Calthion palustris, Magnocaricion elatae, Scheuchzerio-Caricetalia nigrae: Obreja, Sadova Nouă, V. Bistra, Zerveşti (Boşcaiu N., 1971); var. fontanum Hausskn.: Groapa Bistrei în M-ţii Ţarcu, M-ţii Semenic (Flora V)

Epilobium parviflorum Schreber: H, Eua; U5T3R4.5, Glycerio-Sparganion, Phragmitetea: Armeniş (Boşcaiu N., 1971,!), Bucoşniţa (!), Gărâna (!), Lugoj (Boşcaiu N., 1966), Măgura (Boşcaiu N., 1971), Pădureni on Timişul Mort (Soran V., 1956), Poiana Mărului, Turnu Ruieni, V. Bistra, V. Bistra Mărului (Boşcaiu N., 1971), Urseni (Soran V., 1956),

Epilobium roseum Schreber: H, Eua; U4.5T3R4.5, Glycerio-Sparganion: Măgura, Mt. Ţarcu, Poiana Mărului, Turnu Ruieni (Boşcaiu N., 1971)

Epilobium tetragonum L. (E. adnatum Griseb.): H, Eua (sM); U4.5T3R0, Agrostion stoloniferae, Bidentetalia tripartiti, Magnocaricion elatae: Bazoş (Flora V)

Epilobium $\boldsymbol{x}$ boissieri Hausskn. (anagalidifolium x alsinifolium): Mt. Ţarcu la Izvorul Hidegu (Flora V, Boşcaiu N., 1971)

Ludwigia palustris (L.) Elliot: Th-Hh, Atl-M; U4,5T3,5R3,5, Magnocaricion; Lugoj (Boşcaiu N., 1966 ap. J. Heuffel, 1858; Flora V)

\section{Trapaceae}

Trapa natans L. subsp. natans: Hh, Eua-sM; U6T4R4, Nymphaeion: Liebling (Neacşu A. et al., 2008), Lugoj in lakes from Complexul I şi II (Boşcaiu N., 1966)

\section{Euphorbiaceae}

Euphorbia lucida Waldst. et Kit.: H, E-C; U5T3R4, Filipendulo-Petasition, Molinion coeruleae: Lugoj (Flora II)

Euphorbia villosa Walds. Et Kit.: H, p-M; U3,5T3,5R0, Molinion, Alnetea: Caransebeş-Gara Ţiglărie (Boşcaiu N., 1965), between Zerveşti and Turnu Rueni (Boşcaiu N., 1965), Zlagna (Boşcaiu N., 1965) 


\title{
Rhamnaceae
}

Frangula alnus Miller: mPh, Eua; U4T3R3, Alno-Padion, Alnetea: Caransebeş (!) at Gara Ţiglărie (Boşcaiu N., 1965), between Zerveşti and Turnu Rueni (Boşcaiu N., 1965), Zlagna (Boşcaiu N., 1965)

Vitaceae

Vitis silvestris Gmel.: mPh-L, P-M; U3.5T4.5R4.5, Alno-Padion, Querco-Fagetea, Salicion albae: Caransebeş (Flora VI)

\author{
Apiaceae \\ Angelica archangelica L.: TH-H, Eua (bor); U4.5T2.5R0, Adenostyletalia, Filipendulo- \\ Petasition: Armeniş (!) on Râul Alb and Râul Lung (Boşcaiu N., 1971), Borlova under Mt. Mic \\ (Flora VI, Boşcaiu N., 1971), Gărâna (!), Izvorul Sebeşului, Pâr. Hidegu, Poiana Mărului, \\ Rusca, V. Bistra Mărului (Boşcaiu N., 1971), Trei Ape (!) \\ Angelica sylvestris L. ssp. sylvestris: TH-H, Eua; U4T3R3, Alno-Padion, Molinietalia: \\ Armeniş (Boşcaiu N., 1971,!), Bucoşniţa (!), Măgura, Obreja, Pâr. Hidegu, Poiana Mărului \\ (Boșcaiu N., 1971), Petroșnița (!), Sadova Veche (Boșcaiu N., 1966), Trei Ape (!), V. Bistra, \\ V. Bistra Mărului (Boşcaiu N., 1971); f. stenoptera (Boiss.) Thell.: Borlova under Mt. Mic \\ (Flora VI) \\ Angelica $x$ mixta Nyar. (archangelica x sylvestris): Armeniş (!), Gărâna (!) \\ Apium nodiflorum (L.) Lag.: H, Atl-M; U5T4.5R0, Agropyro-Rumicion, Bidention tripartiti, \\ Nanocyperion flavescentis: between Lugoj and Satu Mic (Flora VI ap. J. Heuffel, 1858) \\ Berula erecta (Hudson) Coville (Sium erectum Hudson): H-Hh, Cp; U6T3,5R0, Alno-Padion, \\ Glycerio-Sparganion, Magnocaricion elatae: Caransebeş, Giulvăz, Peciu Nou (!), Pădureni on \\ Timişul Mort (Soran V., 1956), Urseni (Soran V., 1956) \\ Heracleum palmatum Baumg.: H, Carp; U4T2,5R0, Adenosrylion: Mt. Mic, Mt. Țarcu \\ (Boşcaiu N., 1971) \\ Laserpitium prutenicum L.: TH, Ec-M; U4T3,5R4, Molinion: Turnu Ruieni, var. (Boşcaiu N., \\ 1971) \\ Oenanthe aquatica (L.) Poiret: Hh, Eua; U6T3R0, Phragmitetalia: Liebling (Neacşu A. et al., \\ 2008), Pădureni on Timişul Mort (Soran V., 1956), Rudna, Uliuc (Stere G., 1971a), Urseni \\ (Soran V., 1956, Stere G., 1971a) \\ Oenanthe banatica Heuffel: H, P-Pn-B; U4T3.5R0, Alno-Padion: Lugoj (Flora VI) \\ Oenanthe peucedanifolia Pollich (O. stenoloba Schur): H, D-B; U4T0R4.5, Molinietalia: \\ Băuţar (Flora VI, Boşcaiu N., 1971) \\ Oenanthe silaifolia Bieb.: H, M; U5T3.5R0, Agrostion stoloniferae: Armeniş, Glimboca, \\ Obreja, Turnu Ruieni, V. Bistra, Zerveşti (Boşcaiu N., 1971); var. media (Griseb.) Beck: \\ Lugoj (Flora VI) \\ Selinum carvifolia (L.) L.: H, Eua; U3,5T3R3, Molinion: Caransebeş-Gara Ţiglărie (Boşcaiu \\ N., 1965), between Zerveşti and Turnu Rueni (Boşcaiu N., 1965, 1971) \\ Sium latifolium L.: Hh, Eua; U6T0R4, Phragmitetalia, Phragmition australis: Bazoş (Flra VI)

\section{Hypericaceae} \\ Hypericum tetrapterum Fries. (H. quadrangulum L.): H, E; U4T3R4, Filipendulo-Petasition, \\ Glycerio-Sparganion, Magnocaricion elatae: Borlova (Boşcaiu N., 1971), Caransebeş (!), \\ Izvorul Sebeşului, Marga, Măgura, Pâr. Hidegu, Poiana Mărului, Rusca, V. Bistra, V. Bistra \\ Mărului (Boşcaiu N., 1971)
}




\section{Elatinaceae}

Elatine alsinastrum L.: Th-TH (Hh), Eua; U5T4R3, Nanocyperion flavescentis, Potamion: Jebel (Flora III, Soran V., 1956), Liebling (Soran V., 1956)

Elatine hexandra (Lapierre) DC.: Th-TH (Hh), Ec; U5T3R2, Nanocyperion flavescentis: Lugoj (Flora III)

Elatine macropoda Guss. (E. hungarica Moesz): Hh, Eua(M): U5T4R2, Nanocyperion flavescentis: Uliuc, Urseni (Stere G., 1971)

\section{Tamaricaceae}

Myricaria germanica (L.) Desv.: nPh, Eua; U0T0R4.5, Salicion eleagni: Pâr. Hidegu (Boşcaiu N., 1971)

\section{Brassicaceae}

Cardamine amara L. ssp. amara: H, Eua; U5T0R0, Alno-Padion, Cardamini-Montion: Armeniş (!), Borlova (Boşcaiu N., 1971), Gărâna (!), Groapa Bistrei, Marga, Măgura, Mt. Mic (Boșcaiu N., 1971), Mții Semenic (Flora III), Pâr. Hidegu, Poiana Mărului (Boșcaiu N., 1971), Trei Ape (!), V. Bistra Mărului, V. Sebeşului (Boşcaiu N., 1971, 1971a); ssp. opicii (J. et C. Presl.) Celak.: H, Ec: Groapa Bistrei (Flora III, Boşcaiu N., 1971a), Mt. Mic (Boşcaiu N., 1971, 1971a), Izvorul Bistrei Mărului, Vf. Căleanu, Vf. Ţarcu (Boşcaiu N., 1971)

Cardamine flexuosa With.: Th-TH, Eua; U4T2R2, Cardamini-Montion: Armeniş (!) on Râul Alb and Râul Lung, Borlova, Măgura, Pâr. Hidegu, Poiana Mărului, Sadova Nouă, V. Sebeşului (Boşcaiu N., 1971)

Cardamine pratensis L. ssp. pratensis: H, Cp; U5T3R0, Molinio-Arrhenatheretea: Caransebeş-Gara Ţiglărie (Boșcaiu N., 1965), Obreja, Pâr. Hidegu, V. Bistra (Boşcaiu N., 1971), between Zerveşti and Turnu Rueni (Boşcaiu N., 1965, 1971); ssp. matthioli (Moretti) Nyman (ssp. hayneana (Welw.) D.E. Schultz): H, E: Poiana Mărului (Boşcaiu N., 1971); ssp. rivularis (Schur) Nyman: H, Carp-B: Vf. Căleanu (Boşcaiu N., 1971), Vf. Tarcu (Boșcaiu N., 1971, 1971a)

Rorippa amphibia (L.) Besser: Hh, Eua; U6T3R4, Alnetea glutinosae, Phragmitetea, Salicetea purpureae: Liebling (Neacşu A. et al., 2008), Lugoj (Boşcaiu N., 1966,!)

Rorippa austriaca (Crantz) Besser: H, P; U4T3.5R4, Agropyro-Rumicion, Bidentetea tripartiti, Plantaginetea majoris, Senecion fluviatilis: Giulvăz (!), Jebel (Soran V., 1956), Liebling (Neacşu A. et al., 2008) in Balta Mare and Balta Mică (Soran V., 1956)

Rorippa sylvestris (L.) Besser ssp. sylvestris: H, Eua; U4T3R4, Agropyro-Rumicion: Armeniş (Boşcaiu N., 1971,!), Boldur, Bucoşniţa (!), Borlova (Boşcaiu N., 1971), Caransebeş, Giulvăz (!), Ilova (Boşcaiu N., 1971), Liebling (Neacşu A. et al., 2008), Lugoj (Boşcaiu N., 1966), Marga, Măgura, Obreja (Boșcaiu N., 1971), Oțelul Roșu (Boșcaiu N., 1971,!), Rusca, Sadova Veche (Boşcaiu N., 1971), Şag, Trei Ape (!), Turnu Ruieni, V. Bistra Mărului, V. Sebeşului, Zăvoi, Zerveşti (Boşcaiu N., 1971); f. rivularis (Rchb.) Nyar.: Sadova Nouă (Boşcaiu N., 1971 ap. V. Borbas, 1873); f. siliculosa Neilr.: Sadova Nouă (Flora III, Boșcaiu N., 1971 ap. V. Borbas, 1873); ssp. kerneri: Liebling (Soran V., 1954),

Rorippa $x$ barbareoides (Tsch.) Celak. (palustris $\mathbf{x}$ sylvestris). Armeniş (!); f. arenaria Knaf: Armeniş (Flora III)

\section{Salicaceae}

Populus alba L.: Mph-mPh, Eua; U3.5T3R3, Salicetalia purpureae: Grănicerii (Ciavoş) (Flora I) 
Populus nigra L.: MPh, Eua; U4T3R4, Salicetalia purpureae: Armeniş (Boşcaiu N., 1971), Grănicerii (Ciavoş) (Flora I),

Salix alba L. ssp. alba: Mph-mPh, Eua; U5T3R4, Alno-Padion, Salicion albae: Armeniş (Boşcaiu N., 1971,!), Borlova (Boşcaiu N., 1971), Belinţ, Bucoşniţa, Caransebeş, Chizătău (!), Liebling (Neacşu A. et al., 2008), Lugoj (Boşcaiu N., 1966,!), Pâr. Hidegu, Poiana Mărului, Rusca (Boşcaiu N., 1971), Sadova Veche (!), Turnu Ruieni, V. Bistra, V. Bistra Mărului (Boşcaiu N., 1971),

Salix cinerea L.: mPh, Eua; U5T3R3, Alnetea glutinosae, Alno-Padion: Armeniş (Boşcaiu N., 1971,!), Caransebeş-Gara Ţiglărie (Boşcaiu N., 1965), Glimboca (Boşcaiu N., 1971), Liebling (Neacşu A. et al., 2008), Lugoj (Boşcaiu N., 1966), Măgura, Obreja, Poiana Mărului, Sadova Veche (Boşcaiu N., 1971), Trei Ape (!), V. Bistra, V. Bistra Mărului, var. (Boşcaiu N., 1971), between Zervești and Turnu Rueni (Boşcaiu N., 1965, 1971)

Salix daphnoides Vill.: mPh, Eua; U4.5T2.5R4.5, Salicion eleagni: V. Bistra beside Măgura (Boşcaiu N., 1971)

Salix fragilis L.: mPh-MPh, Eua; U4.5T3R4, Alno-Padion, Salicion albae, Salicion triandrae: Armeniş, Borlova (Boşcaiu N., 1971), Bucoşniţa, Caransebeş (!), Liebling (Neacşu A. et al., 2008), Pâr. Hidegu, Rusca (Boşcaiu N., 1971), Trei Ape (!), Turnu Ruieni, V. Bistra, V. Bistra Mărului (Boşcaiu N., 1971)

Salix purpurea L. ssp. purpurea: $\mathrm{mPh}$, Eua; U5T3R4.5, Salicetalia purpureae: Armeniş (Boşcaiu N., 1971, !), Borlova, Izvorul Sebeşului (Boşcaiu N., 1971), Caransebeş (!), Lugoj (Boşcaiu N., 1966), Pâr. Hidegu, Măgura, Rusca, Turnu Ruieni, V. Bistra, V. Bistra Mărului (Boşcaiu N., 1971), Sadova Veche (Boşcaiu N., 1971, !)

Salix triandra L. emend. Ser. ssp. triandra: mPh, Eua; U5T3R0, Salicion triandrae: Armeniş (!) pe Râul Lung, Borlova, Izvorul. Sebeşului (Boşcaiu N., 1971), Lugoj (Boşcaiu N., 1966), Măgura, Obreja, Pâr. Hidegu, Rusca (Boşcaiu N., 1971), Trei Ape (!) Turnu Ruieni, V. Bistra, V. Bistra Mărului, Zervești (Boşcaiu N., 1971)

Salix viminalis L.: mPh, Eua; U5T2.5R4.5, Salicion triandrae: Măgura on Bistra (Boşcaiu N., 1971)

Salix $x$ rubens Schrank. (alba x fragilis): Armeniş on Râul Alb, Borlova, Glimboca, Măgura, Turnu Ruieni (Boşcaiu N., 1971)

Salix $x$ undulata Ehrh. (alba x triandra): Măgura on Bistra (Boşcaiu N., 1971)

Cucurbitaceae

Sicyos angulatus L.: Th, Adv; U4.5T3R4, Calystegion: Lugoj (Boşcaiu N., 1966)

\section{Primulaceae}

Lysimachia nummularia L.: Ch, Eua; U4T3R0, Alnetea glutinosae, Alno-Padion, Bidentetea tripartiti, Calthion palustris, Filipendulo-Petasition, Molinietalia, Phragmitetea, Plantaginetea majoris, Querco-Fagetea, Salicion albae: Armeniş (Boşcaiu N., 1971,!), Belinţ (!), Borlova (Boşcaiu N., 1971), Caransebeş, Giulvăz (!), Glimboca, Ilova (Boşcaiu N., 1971), Liebling (Neacşu A. et al., 2008), Lugoj (Boşcaiu N., 1966,!), Măgura, Obreja (Boşcaiu N., 1971), Oţelul Roşu (Boşcaiu N., 1971,!), Pâr. Hidegu, Poiana Mărului, Râul Alb, Rusca, Sadova Veche, Turnu Ruieni, V. Bistra, V. Bistra Mărului, Var, Vălişoara, Vârciorova, Zerveşti, Zlagna, (Boşcaiu N., 1971), Trei Ape (!) 
Lysimachia vulgaris L.: H(-Hh), Eua; U5T0R0, Alnetea glutinosae, Molinietalia, Phragmitetea, Salicetea purpureae, Scheuchzerio-Caricetea nigrae: Armeniş (Boşcaiu N., 1971,!), Borlova (Boşcaiu N., 1971), Caransebeş (!) at Gara Ţiglărie (Boşcaiu N., 1965), Glimboca (Boşcaiu N., 1971), Liebling (Neacşu A. et al., 2008), Lugoj (!), Marga, Măgura, (Boşcaiu N., 1971), Oțelul Roşu (Boşcaiu N., 1971, !), Pâr. Hidegu (Boşcaiu N., 1971), Petroşniţa (!),Poiana Mărului, Rusca, Sadova Veche (Boşcaiu N., 1971), Tapia (Boşcaiu N., 1966), V. Bistra, V. Bistra Mărului, (Boşcaiu N., 1971), Trei Ape (!), between Zerveşti and Turnu Rueni (Boşcaiu N., 1965)

\section{Gentianaceae}

Centaurium pulchellum (Swartz) Druce: Th-TH, Eua; U4T3.5R4, Isoeto-Nanojuncetea: Buziaş, Lugoj (Flora VIII), Obreja, V. Bistra, var. (Boşcaiu N., 1971)

Gentiana pneumonanthe L.: H, Eua; U4T3R0, Molinion: Caransebeş-Gara Ţiglărie (Boşcaiu N., 1965), Sadova Veche (Boşcaiu N., 1971) between Zerveşti and Turnu Rueni (Boşcaiu N., 1965, 1971), Zlagna (Boşcaiu N., 1965, 1971)

\section{Solanaceae}

Solanum dulcamara L.: Ch (nPh), Eua; U4.5T3R4, Alnetea glutinosae, Alno-Padion, Bidentetea tripartiti, Calystegion, Epilobietalia angustifolii, Phragmition australis: Caransebeş (!), Liebling (Neacşu A. et al., 2008), Lugoj (Boşcaiu N., 1966,!), Pădureni on Timişul Mort (Soran V., 1956), Trei Ape (!), Urseni (Soran V., 1956)

\section{Convolvulaceae}

Calystegia sepium (L.) R.Br.: G(H), Eua; U4,5T3R4, Calystegion, Salicion albae, Arction lappae: Armeniş (Boşcaiu N., 1971, !), Borlova (Boşcaiu N., 1971), Bucoşniţa (!), Caransebeş, Giulvăz (!), Glimboca (Boşcaiu N., 1971), Liebling (Neacşu A. et al., 2008), Lugoj (Boşcaiu N., 1966), Măgura, Obreja (Boşcaiu N., 1971), Oțelul Roşu (Boşcaiu N., 1971,!), Petroşnița (!), Rusca, Sadova Veche, Turnu Ruieni, V. Bistra, Var, Zerveşti, (Boşcaiu N., 1971)

\section{Menyanthaceae}

Menyanthes trifoliata L.: Hh, Cp; U5T0R0, Magnocaricion elatae, Scheuchzerio-Caricetalia nigrae: Lugoj (Boşcaiu N., 1966 ap. J. Heuffel, 1858)

\section{Boraginaceae}

Myosotis scorpioides L. (M. palustris (L.) Hill): H(Hh), Eua; U5T3R0, Alnetea glutinosae, Calthion palustris, Molinietalia, Phragmitetea: Caransebeş (!) at Gara Ţiglărie (Boşcaiu N., 1965), Liebling (Neacşu A. et al., 2008), Marga, Măgura, Obreja, Pâr. Hidegu, Rusca, (Boşcaiu N., 1971), Trei Ape (!), Var, (Boşcaiu N., 1971), between Zerveşti and Turnu Rueni (Boşcaiu N., 1965, 1971); var. elatior Opiz: Mt. Semenic (Flora VII)

Symphytum officinale L. ssp. officinale: H, Eua; U4T3R0, Molinietalia, Phragmitetea: Armeniş (Boşcaiu N., 1971, !), Borlova (Boşcaiu N., 1971), Caransebeş, Giulvăz (!), Glimboca, Ilova (Boşcaiu N., 1971), Liebling (Neacşu A. et al., 2008), Lugoj, Tapia (Boşcaiu N., 1966,!), Marga, Măgura, Pâr. Hidegu (Boşcaiu N., 1971), Oţelul Roşu (Boşcaiu N., 1971,!), Petroşniţa (!), Rusca, Sadova Veche (Boşcaiu N., 1971), Trei Ape (!)Turnu Ruieni, V. Bistra, V. Bistra Mărului, V. Sebeşului, Zerveşti (Boşcaiu N., 1971) 
Lamiaceae

Lycopus europaeus L.: H (Hh), Eua; U5T3R0, Bidentetea tripartiti, Phragmitetea, Salicetea purpureae: Armeniş (Boșcaiu N., 1971,!), Borlova (Boșcaiu N., 1971), Caransebeş, Gărâna (!), Glimboca, Ilova (Boşcaiu N., 1971), Liebling (Neacşu A. et al., 2008), Lugoj (Boşcaiu N., 1966,!), Oțelul Roşu (Boşcaiu N., 1971,!), Obreja, Pâr. Hidegu, Rusca (Boşcaiu N., 1971), Pădureni on Timişul Mort (Soran V., 1956), Petroşniţa (!), Tapia (Boşcaiu N., 1966), Teregova (!), Trei Ape (!), Turnu Ruieni, V. Bistra, V. Bistra Mărului, Zerveşti, (Boșcaiu N., 1971)

Lycopus exaltatus L. fil: H(Hh), Eua-C; U5T3R0, Bidentetea tripartiti, Phragmitetea, Salicion albae: Lugoj in the lake from Str. Bocșei (Boșcaiu N., 1966)

Mentha aquatica L.: H (Hh), E; U5T3R0, Alnetea glutinosae, Molinietalia, Phragmitetea, Salicion albae: Armeniş (Boşcaiu N., 1971,!), Caransebeş (!), Liebling (Neacşu A. et al., 2008), Obreja (Boşcaiu N., 1971), Urseni (Soran V., 1956), V. Bistra Mărului, Zervești, (Boşcaiu N., 1971); var. stagnalis Top.: Liebling (Flora VIII)

Mentha arvensis $\mathbf{L}$. ssp. arvensis (ssp. agrestis (Sole) Briq.): H(G), Cp; U4T3R0, Calthion palustris, Molinietalia, Phragmitetea, Secalietea: Armeniş (Boșcaiu N., 1971,!), Gărâna (!), Izvorul Sebeşului, Marga, Obreja (Boşcaiu N., 1971), Oțelul Roşu (Boşcaiu N., 1971,!), Trei Ape (!), Zerveşti, (Boşcaiu N., 1971)

Mentha longifolia (L.) Hudson ssp. longifolia: H(G), Eua; U4,5T3R0, Agropyro-Rumicion, Bidentetea tripartiti, Chenopodietea, Filipendulo-Petasition, Glycerio-Sparganion, Molinietalia: Armeniş (Boşcaiu N., 1971,!), Bolvaşniţa, Borlova (Boşcaiu N., 1971), Bucoşnița (!), Gărâna, Giulvăz (!), Ilova (Boşcaiu N., 1971), Oțelul Roşu (!), Petroşniţa (!), Pâr. Hidegu, Poiana Mărului, Rusca (Boşcaiu N., 1971), Sadova Nouă, Sadova Veche (Boşcaiu N., 1971), Şag, Teregova (!), Trei Ape (!), Turnu Ruieni, V. Bistrei, V. Bistra Mărului, V. Sebeșului, var. (Boșcaiu N., 1971)

Mentha pulegium L.: H, Eua (sM); U4T3R5, Isoeto-Nanojuncetea, Nanocyperion flavescentis: Armeniş (Boşcaiu N., 1971,!), Caransebeş (!), Cruceni, Foeni (Stere G., 1971a), Giulvăz (!), Ivanda (Stere G., 1971a), Jebel, Liebling (Soran V., 1956), Lugoj (!) beside Complexul I and V. Cinca beside Boldur (Boșcaiu N., 1966), Obreja, (Boșcaiu N., 1971), Oțelul Roşu (Boşcaiu N., 1971,!), Petroşniţa (!), Pâr. Hidegu (Boşcaiu N., 1971), Rudna (Stere G., 1971a), Rusca, Sadova Veche (Boşcaiu N., 1971), Şag, Teregova (!), Turnu Ruieni (Boşcaiu N., 1971), Urseni (Stere G., 1971a), V. Bistrei, V. Bistra Mărului, Var, Zerveşti, (Boşcaiu N., 1971)

Mentha $x$ verticillata $\mathrm{L}$. (aquatica $\mathrm{x}$ arvensis): V. Ştiuca beside Tapia locality (Boşcaiu N., 1966)

Scutellaria galericulata L.: H, Cp; U4T3R4, Magnocaricion elatae, Molinietalia, Phragmitetea: Glimboca (Boşcaiu N., 1971,!), Liebling (Neacşu A. et al., 2008), Lugoj (Boşcaiu N., 1966), Marga, Obreja, Oţelul Roşu (Boşcaiu N., 1971), Trei Ape (!), V. Bistrei, Zerveşti (Boşcaiu N., 1971)

Stachys palustris: Armeniş (Boşcaiu N., 1971,!), Bucoşniţa (!), Caransebeş (!), Liebling (Neacşu A. et al., 2008), Lugoj at the brick yard Bartoş (Boşcaiu N., 1966), Obreja (Boşcaiu N., 1971), Oţelul Roşu (Boşcaiu N., 1971,!), Sadova Veche (Boşcaiu N., 1971), Trei Ape (!), Zervești (Boşcaiu N., 1971) 


\section{Callitrichaceae}

Callitriche cophocarpa Sendtner (C. polymorpha Lonnr.): Th-H (Hh), Eua; U6T3R0, Nanocyperion flavescentis, Potamion: Caransebeș-Gara Țiglărie (Boșcaiu N., 1965), Glimboca (Flora II, Boşcaiu N., 1971), Lugoj (Flora II), Obreja (Flora II, Boşcaiu N., 1971)

Callitriche palustris L. (C. verna L.): Th-H (Hh), Cp; U6T3R0, Nanocyperion flavescentis, Potamion: Mt. Mic (Boşcaiu N., 1971, 1971a), Mt. Ţarcu, Pâr. Hidegu, Poiana Mărului, Râul Alb, Râul Lung (Boşcaiu N., 1971), Rusca (Flora II, Boşcaiu N., 1971), Trei Ape (!), V. Bistra Mărului (Boşcaiu N., 1971)

\section{Scrophulariaceae}

Gratiola officinalis L.: H, Cp; U4.5T3R4, Magnocaricion elatae, Molinion coeruleae, Nanocyperetalia, Phragmitetea: Caransebeş-Gara Ţiglărie (Boşcaiu N., 1965), Jebel (Soran V., 1956), Liebling (Neacşu A. et al., 2008, Soran V., 1956), Lugoj (Boşcaiu N., 1966), between Zerveşti and Turnu Rueni (Boşcaiu N., 1965)

Lindernia procumbens (Krocker) Philcox (L. pyxidaria L. p.p.): Th, Eua; U4.5T4R0, Nanocyperion flavescentis: Jebel, Liebling (Soran V., 1954, 1956, Flora VII), Lugoj (Boșcaiu N., 1966, Flora VII), Obreja (Boșcaiu N., 1971), Pădureni (Flora VII), Urseni (Soran V., 1954)

Scrophularia umbrosa Dumort: H, Eua; U5T3,5R4,5, Alno-Padion, Glycerio-Sparganion: Armeniş, Pâr. Hidegu (Boşcaiu N., 1971), Urseni (Soran V., 1956)

Veronica anagallis-aquatica L.: H (Hh), Cp; U5T0R4, Bidentetea tripartiti, GlycerioSparganion, Phragmitetea : Bucoşniţa (!), Teregova (Boşcaiu N., 1971), V. Sebeşului (Boşcaiu N., 1971a)

Veronica beccabunga L.: H (Hh), Eua; U5T3R4, Bidentetea tripartiti, GlycerioSparganion, Salicetalia purpureae: Armeniş (!), Mt. Ţarcu (Boşcaiu N., 1971a), Măgura, Pâr. Hidegu, Poiana Mărului (Boșcaiu N., 1971), Trei Ape (!), V. Bistra Mărului (Boșcaiu N., 1971)

Veronica scutellata L.: H (Hh), Cp; U4T3R4, Agrostion stoloniferae, Caricion canescentinigrae, Magnocaricion elatae: Lugoj (Flora VII), Trei Ape (!)

Tozzia alpina L. ssp. carpatica (Woloszczak) Hayek: H, Carp-B; U3.5T2R4.5, Adenostyletalia, Cardamini-Montion: Mţii Semenic, Mt. Ţarcu în V. Bistra (Flora VII, Boşcaiu N., 1971)

\section{Lentibulariaceae}

Utricularia vulgaris L.: Hh, Cp; U6T0R3,5, Nymphaeion, Lemnion-Hydrocharition: Belinţ, Chizătău, Foeni (Stere G., 1971a), Lugoj in the lake in Str. Bocşei and at the brick yard Bartoş (Boşcaiu N., 1966), Pădureni on Timişul Mort and in puddles (Soran V., 1956), Rudna, Uliuc, Urseni (Stere G., 1971a)

\section{Rubiaceae}

Galium palustre L. ssp. palustre: H, Cp; U5T3R0, Magnocaricion elatae, Molinietalia: Armeniş (Boşcaiu N., 1971,!), Caransebeş (!) at Gara Țiglărie (Boşcaiu N., 1965), Glimboca (Boşcaiu N., 1971), Lugoj (Boşcaiu N., 1966,!), Marga, Măgura, Pâr. Hidegu, Obreja, (Boşcaiu N., 1971) Trei Ape (!), between Zerveşti and Turnu Rueni (Boşcaiu N., 1965, 1971)

Galium uliginosum L.: H, Eua; U4.5T3R4, Calthion palustris, Magnocaricion elatae, Molinietalia, Scheuchzerio-Caricetalia nigrae: Armeniş, Marga (Boşcaiu N., 1971), Mt. Mic (Boşcaiu N., 1971a), Obreja, Rusca, Turnu Ruieni, V. Bistra Mărului, var. (Boşcaiu N., 1971) 


\begin{abstract}
Valerianaceae
Valeriana officinalis L.: H, Eua(sM); U4T3R4, Alnetea glutinosae, Alno-Padion, FilipenduloPetasition, Magnocaricion elatae, Molinietalia; Caransebeș-Gara Țiglărie (Boșcaiu N., 1965), between Zerveşti and Turnu Rueni (Boşcaiu N., 1965); f. altissima (Hornem.) Koch: Armeniş, Borlova, Izvorul Sebeşului, Măgura, Oțelul Roşu, Pâr. Hidegu, Poiana Mărului, Rusca, Turnu Ruieni, V. Bistra Mărului, Zărneşti (Boşcaiu N., 1971 under V. exaltata Mikan)

Valeriana sambucifolia Mikan fil. (V. officinalis L. ssp. sambucifolia (Mikan fil.) Celak.): H, Ec; U4T2R3.5, Adenostyletalia, Fagion, Filipendulo-Petasition: Izvorul Sebeşului, Mt. Mic, Poiana Mărului, V. Bistra Mărului (Boşcaiu N., 1971)

Dipsacaceae

Succisa pratensis Moench: H, Eua; U4T3R0, Molinietalia, Molinion coeruleae: Borlova (Boşcaiu N., 1971), Caransebeş (!) at Gara Ţiglărie (Boşcaiu N., 1965), Feneş (Boşcaiu N., 1971), Gărâna (!), Lugoj, in V. Ştiuca beside Tapia (Boşcaiu N., 1966), Marga, Obreja, Oțelul Roşu, Pâr. Hidegu (Boşcaiu N., 1971), Trei Ape (!), between Zerveşti and Turnu Rueni (Boşcaiu N., 1965), Zlagna (Boşcaiu N., 1965)
\end{abstract}

\title{
Asteraceae
}

Achillea ptarmica L.: H, Eua; U4,5T0R2,5, Molinietalia: Borlova (Boşcaiu N., 1971), Caransebeş-Gara Țiglărie (Boşcaiu N., 1965), Obreja, Oţelul Roşu, Poiana Mărului, V. Bistra, V. Bistra Mărului, V. Sebeşului (Boşcaiu N., 1971)

Aster $x$ salignus Willd. (lanceolatus x novi belgii): Liebling (Flora IX)

Bidens cernua L.: Th, Eua; U5T0R0, Bidention: Râul Lung, V. Bistra (Boşcaiu N., 1971)

Bidens tripartita L.: Th, Eua; U4.5T3R0, Bidentetea tripartiti, Chenopodio-Scleranthetea, Nanocyperion flavescentis: Armeniş (Boşcaiu N., 1971,!), Bucoşniţa (!), Caransebeş, Giulvăz (!), Ilova (Boşcaiu N., 1971), Liebling (Neacşu A. et al., 2008), Lugoj (Boşcaiu N., 1966,!), Obreja (Boşcaiu N., 1971), Petroşniţa (!), Teregova (!), Rusca, Sadova Veche (Boşcaiu N., 1971,!), Trei Ape (!), V. Bistra, V. Sebeşului (Boşcaiu N., 1971)

Bidens vulgata E. L. Greene: Th, Adv; U5T0R0, Bidention: Armeniş (!), Bucoşniţa (!), Sadova Veche (!), Criciova (Flora XIII)

Carduus personatus (L.) Jacq. ssp. personatus: H, Ec; U4.5T2.5R4.5, Adenostylion alliariae, Alno-Padion, Filipendulo-Petasition: Borlova, Groapa Bistrei, Mt. Mic, Pâr. Hidegu, Poiana Mărului, Râul Alb, Râul Lung (Boşcaiu N., 1971), Trei Ape (!), V. Bistra, V. Bistra Mărului, V. Sebeşului (Boşcaiu N., 1971)

Cirsium brachycephalum Juratzka: TH, Pn; U4T3R4, Agrostion stoloniferae, Magnocaricion elatae: Moşniţa (Flora IX)

Cirsium canum (L.) All.: G, Eua-C; U4.5T3R4.5, Alno-Padion, Magnocaricion elatae, Molinietalia: Armeniş (Boşcaiu N., 1971, !), Caransebeş-Gara Ţiglărie (Boşcaiu N., 1965), Glimboca (Boşcaiu N., 1971, !), Liebling (Neacşu A. et al., 2008), Obreja, Oţelul Roşu, Pâr. Hidegu, Sadova Veche, V. Bistra (Boşcaiu N., 1971)

Cirsium erisithales (Jacq.) Scop.: H, Ec (mont); U3,5T3R4.5, Fagetalia silvaticae, Filipendulo-Petasition: Groapa Bistrei, Pâr. Hidegu, Poiana Mărului, Râul Alb, Râul Lung, Rusca, V. Bistra Mărului, V. Sebeşului (Boşcaiu N., 1971)

Cirsium oleraceum (L.) Scop.: H, Eua; U4T3R4, Alno-Padion, Calthion palustris, Filipendulo-Petasition, Molinietalia: Armeniş (!) pe Râul Lung, Pâr. Hidegu, Poiana Mărului, V. Bistra Mărului, Voislova (Boşcaiu N., 1971) 
Cirsium palustre (L.) Scop.: TH, Eua; U4.5T3R2.5, Alnetea glutinosae, Epilobietalia angustifolii, Molinietalia, Phragmitetea: Gărâna (!), Liebling (Neacşu A. et al., 2008), Pâr. Hidegu, Poiana Mărului, Râul Alb, Râul Lung, Rusca, Sadova Nouă (Boşcaiu N., 1971), Trei Ape (!), V. Bistra, V. Bistra Mărului, V. Sebeşului, Zlagna (Boşcaiu N., 1971)

Cirsium waldsteinii Rouy: H, Alp-Carp; U4T2R2, Adenostylion alliariae, Caricion curvulae, Filipendulo-Petasition: Poiana Mărului (Boşcaiu N., 1971)

Crepis paludosa (L.) Moench: H, E (mont); U4.5T0R4.5, Adenostyletalia, Alnetea glutinosae, Alno-Padion, Calthion palustris, Montio-Cardaminetea: Armeniş on Râul Alb and Râul Lung, Pâr. Hidegu, Poiana Mărului, Sadova Nouă, V. Bistra, V. Bistra Mărului (Boşcaiu N., 1971), V. Sebeşului (Boşcaiu N., 1971, 1971a), Trei Ape (!)

Eupatorium cannabinum L.: H, Eua; U4T3R0, Alnion glutinosae, Epilobietea angustifolii, Filipendulo-Petasition, Phragmitetea, Salicetalia purpureae: Armeniş (!) on Râul Alb and Râul Lung, Borlova (Boşcaiu N., 1971), Bucoşniţa, Caransebeş (!), Liebling (Flora IX), Lugoj (Boşcaiu N., 1966,!), Mt. Semenic (Flora IX), Marga, Măgura, Obreja (Boşcaiu N., 1971), Pădureni (Flora IX), Petroşniţa (!), Pâr. Hidegu, Poiana Mărului, Rusca (Boşcaiu N., 1971), Sadova Veche (!) Turnu Ruieni, V. Bistra, V. Bistra Mărului, V. Sebeșului (Boșcaiu N., 1971)

Gnaphalium uliginosum L.: Th, Eua; U5T3R4, Nanocyperetalia: Armeniş, Glimboca (Boşcaiu N., 1971), Lugoj (Boşcaiu N., 1966), Măgura, Obreja, Oţelul Roşu, Pâr. Hidegu, Rusca, Turnu Ruieni, V. Bistra Mărului, Voislova, Zerveşti (Boşcaiu N., 1971)

Helianthus decapetalus L.: H, Adv; U3.5T3R4.5, Calystegion, Salicion albae: Bucoşniţa (!), Petroşniţa (!), Sadova Veche (!)

Helianthus tuberosus L.: G, Adv; U3.5T3R4, Calystegion: Uliuc, Urseni (Grigore Stere, 1971) Petasites albus (L.) Gaertner: G, Eua; U3.5T0R0, Alno-Padion, Fagion: Pâr. Hidegu, Rusca (Boşcaiu N., 1971), V. Sebeşului (Boşcaiu N., 1971a)

Petasites hybridus (L.) P. Gaertner, B. Meyer et Scherb.: G, Eua; U5T3R3, Adenostyletalia, Alno-Padion, Filipendulo-Petasition: Armeniş (!) on Râul Alb and Râul Lung, Borlova, Pâr. Hidegu, Poiana Mărului, Rusca (Boşcaiu N., 1971), Trei Ape (!), Turnu Ruieni, V. Bistra, V. Bistra Mărului, V. Sebeşului (Boşcaiu N., 1971)

Petasites kablikianus (L.) Tausch.: G, Carp-B; U4T0R0, Galio-Urticetea: Poiana Mărului, V. Bistra Mărului (Boşcaiu N., 1971)

Pulicaria dysenterica (L.) Bernh.: H, Ec; U4T3.5R0, Agropyro-Rumicion, Molinietalia: Armeniş (Boşcaiu N., 1971, !), Caransebeş (!), Cruceni, Foeni (Stere G., 1971a), Giulvăz (Stere G., 1971a,!), Ivanda (Stere G., 1971a), Ilova (Boşcaiu N., 1971), Liebling (Flora IX), Lugoj (Boşcaiu N., 1966, !), Obreja (Boşcaiu N., 1971), Rudna (Stere G., 1971a), Şag, Teregova (!), Urseni (Stere G., 1971a)

Pulicaria vulgaris Gaertner: Th, Eua; U4T3R3, Agropyro-Rumicion, Bidention tripartiti, Isoeto-Nanojuncetea: Armeniş (Boşcaiu N., 1971,!), Bucova (Flora IX), Liebling (Flora IX), Lugoj (Boşcaiu N., 1966), Obreja (Boşcaiu N., 1971), Oţelul Roşu (Boşcaiu N., 1971,!), Zerveşti (Boşcaiu N., 1971)

Rudbeckia laciniata L.: H, Adv; U4.5T3.5R4, Calystegion, Senecion fluviatilis: Bucoşniţa (!), between Lugoj and Careansebeş (Flora IX)

Serratula tinctoria L.: H, Eua; U3.5T3R0, Molinion coeruleae: Caransebeş-Gara Ţiglărie (Boşcaiu N., 1965), Faţa Sadovei (Boşcaiu N., 1965, 1971), Lugoj (Boşcaiu N., 1966), Obreja, Pâr. Hidegu, Rusca, Sadova Nouă, Sadova Veche, Vălişoara (Boşcaiu N., 1971), between Zerveşti and Turnu Rueni (Boşcaiu N., 1965, 1971), Zlagna (Boşcaiu N., 1965, 1971); var. lancifolia S. F. Gray: Bucova (Flora IX), Liebling (Flora IX)

Sonchus palustris L.: G, E; U4.5T3.5R4, Calystegion, Filipendulo-Petasition: Liebling, Pădureni, Timişul Mort (Flora X) 
Taraxacum bessarabicum (Hornem.) Hand.-Mazz.: H, Eua-C; U4T3R4, Puccinellietalia: Jebel, Liebling (Flora IX)

Telekia speciosa (Schreber) Baumg.: H, Carp-B-Cauc-Anat; U4T2R0, Alnion glutinosaeincanae, Filipendulo-Petasition, Telekion: Armeniş (Boşcaiu N., 1971,!), Borlova (Boşcaiu N., 1971), Gărâna (!), Mt. Țarcu, Pâr. Hidegu, Poiana Mărului, Rusca, V. Bistra, V. Bistra Mărului, V. Sebeşului, Zerveşti (Boşcaiu N., 1971)

\begin{abstract}
Alismataceae
Alisma lanceolatum Wither: Hh, Eua; U6T0R4, Phragmitetea: Liebling (Neacşu A. et al., 2008), Moşniţa (Flora XI)

Alisma plantago-aquatica L.: Hh, Cp; U6T0R0, Phragmitetea: Armeniş (Boşcaiu N., 1971,!), Borlova (Boşcaiu N., 1971), Bucoşniţa, Caransebeş (!),Diniaş (Stere G., 1971a), Giulvăz (Stere G., 1971a,!), Glimboca (Boşcaiu N., 1971,!), Jebel, Liebling in Balta Mare and Balta Mică (Soran V., 1956), Lugoj at the brick yard Bartoş (Boşcaiu N., 1966), Oțelul Roşu, Pâr. Hidegu (Boşcaiu N., 1971), Pădureni on Timişul Mort and in puddles (Soran V., 1956), Peciu Nou (Stere G., 1971a,!), Rusca (Boşcaiu N., 1971), Tapia (Boşcaiu N., 1966), Trei Ape (!), Turnu Ruieni (Boşcaiu N., 1971), Urseni (Stere G., 1971a), V. Bistra, V. Bistra Mărului, Var, Zerveşti (Boşcaiu N., 1971)

Şagittaria Şagittifolia L.: Hh, Eua; U6T3R4, Phragmition australis, Potamion: Moşniţa, Urseni (Flora XI), Tapia in V. Ştiuca (Boşcaiu N., 1966)
\end{abstract}

\title{
Butomaceae
}

Butomus umbellatus L.: Hh, Eua; U6T3R0, Phragmitetea: Liebling (Neacşu A. et al., 2008), Lugoj (Boşcaiu N., 1966)

\section{Hydrocharitaceae}

Hydrocharis morsus-ranae L.: Hh, Eua; U6T3.5R3.5, Hydrocharition, Lemnion minoris: Chizătău, Cruceni, Giroc, Ivanda (Stere G., 1971a), Liebling the fen Tofaia (Soran V., 1956), Lugoj in the lake in Str. Bocşei and at the brick yard Bartoş (Boşcaiu N., 1966), Moşniţa, Pădureni (Flora XI) on Timişul Mort (Soran V., 1956), Uliuc, Urseni (Stere G., 1971a)

Stratiotes aloides L.: Hh, Eua; U6T4R4, Hydrocharition, Lemnion minoris: Moşniţa (Flora XI), Uliuc (Stere G., 1971a)

\section{Juncaginaceae}

Triglochin palustris L.: H, Cp; U5T0R0, Molinietalia, Puccinellio-Salicornietea, ScheuchzerioCaricetalia nigrae: Moşniţa (Flora XI)

\section{Potamogetonaceae}

Potamogeton berchtoldii Fieber: Hh, Eua(sM); UTR, U6T3R4, Potamion: Caransebeş (FRE, 1946)

Potamogeton compressus L.: Hh, Cp; U6T3R4, Potamion: Lugoj in the lake in Str. Bocşei (Boşcaiu N., 1966)

Potamogeton crispus L.: Hh, Cosm; U6T3.5R4, Potametalia: Liebling (Neacşu A. et al., 2008) in Balta Mare and Balta Mică (Soran V., 1956), Lugoj at the brick yard Bartoş and in lakes at Complexul III (Boşcaiu N., 1966), between Şag and Urseni (Soran V., 1956)

Potamogeton lucens L.: Hh, Eua; U6T0R4, Potamion: Lugoj, the lake in Str. Bocşei (Boşcaiu N., 1966) 
Potamogeton natans L.: Hh, Cp; U6T2.5R4, Nymphaeion, Potamion: Bazoş (Stere G., 1971a,!), Chizătău, Cruceni (Stere G., 1971a), Costeiu (Flora XI), Liebling (Flora XI, Neacşu A. et al., 2008) in Balta Mare and Balta Mică (Soran V., 1956), Lugoj, lake in Str. Bocşei and at the brick yard Bartoş (Boşcaiu N., 1966), between Lugoj and Tapia on Pâr. Ştiuca (Boşcaiu N., 1966), Peciu Nou, Rudna, Şag (Stere G., 1971a), Urseni (Flora XI)

Potamogeton nodosus Poiret (P. fluitans Roth): Hh, Cp; U6T3.5R4, Potametalia: Cruceni, Diniaş, Foeni (Stere G., 1971a), Lugoj (Boşcaiu N., 1966), Moşniţa Veche, Rudna (Stere G., 1971a)

Potamogeton obtusifolius Mert. et Koch: Hh, Cp; U6T3R3.5, Potamion: Lugoj, lake in Str. Bocşei (Boşcaiu N., 1966), Obreja (Flora XI, Boşcaiu N.,1971); f. elongatum Cham. et Schl.: Obreja (Flora XI)

Potamogeton pectinatus L.: Hh, Cosm; U6T3,5R4,5, Potamion: Liebling in Balta Mare and Balta Mică (Soran V., 1956)

Potamogeton perfoliatus L.: Hh, Cosm; U6T0R4, Potamion: Lugoj in the lakes at Complexul III (Boşcaiu N., 1966)

Potamogeton pusillus L.: Hh, Cosm; U6T3R4, Potamion: Liebling (Flora XI) in Balta Mare (Soran V., 1956), Lugoj (Flora XI); f. acuminatum Fieb.: Lugoj (Flora XI), Otelec, Uliuc (Grigore Stere, 1971)

\section{Najadaceae}

Najas marina L. ssp. marina: Hh (Th), Cosm; U6T4R4, Potamion, Ruppion: Lugoj (Flora XI)

Najas minor All: Hh, Eua; U6T4,5R4,5, Potamion: Lugoj, Lugojel (Boşcaiu N., 1966 ap. J. Heuffel, 1858), Liebling (Flora XI) in Balta Mare and Balta Mică (Soran V., 1956), Lugoj, Pădureni, Slatina (Flora XI), between Şag and Urseni (Soran V., 1956)

\section{Zannichelliaceae}

Zannichellia palustris L. ssp. pedicellata (Wahlenb et Rosen.) Arcang.: Hh, Cosm; U6T0R4, Potamion: V. Cinca beside Boldur (Boşcaiu N., 1966), Liebling (Flora XI) in Balta Mare and Balta Mică (Soran V., 1956), Pădureni (Flora XI), Rudna (Stere G., 1971a), Uliuc (Stere G., 1971)

\section{Liliaceae}

Fritillaria meleagris L.: G, E; U4T3.5R4, Agrostion stoloniferae, Calthion palustris: Buziaş, Liebling (Flora XI)

Veratrum album L.: G, Eua; U4T2,5R4, Molinion, Adenostyletalia: Caransebeş-Gara Ţiglărie (Boşcaiu N., 1965), Mt. Mic, Mt. Țarcu, Obreja, Pâr. Hidegu, Poiana Mărului, Râu Lung, V. Sebeşului (Boşcaiu N., 1971), between Zerveşti and Turnu Rueni (Boşcaiu N., 1965, 1971)

\section{Amaryllidaceae}

Narcissus poëticus L. ssp. radiiflorus (Salisb.) Baker (N. radiiflorus Salisb., N. angustifolius Curt. N. stellaris Haw.): G, Ec; U3.5T2.5R0, Cynosurion cristati, Molinietalia, TrisetoPolygonion: Caransebeş-Gara Ţiglărie (Boşcaiu N., 1965), Faţa Sadovei (Boşcaiu N., 1965, 1971), between Zerveşti and Turnu Rueni (Boşcaiu N., 1965, 1971), Zlagna (Boşcaiu N., 1965, 1971) 


\section{Iridaceae}

Gladiolus imbricatus L.: G, Eua-C; U3,5T3R3, Alno-Padion: Borlova (Boşcaiu N., 1971), Caransebeș-Gara Țiglărie (Boșcaiu N., 1965), Obreja, Pâr. Hidegu, Rusca, V. Bistra, (Boșcaiu N., 1971), between Zerveşti and Turnu Rueni (Boşcaiu N., 1965, 1971)

Iris pseudacorus L.: G (Hh), E; U5.5T0R0, Alnetea glutinosae, Phragmitetea: Lugoj in V. Ştiuca beside Tapia (Boşcaiu N., 1966), Glimboca (Boşcaiu N., 1971), Obreja, Turnu Ruieni (Boşcaiu N., 1971), Urseni (Soran V., 1956), Var, Zerveşti (Boşcaiu N., 1971)

Iris sibirica L.: G, Eua(C); U4,5T3,5R4,5, Molinion: Caransebeş-Gara Ţiglărie (Boşcaiu N., 1965), between Zerveşti and Turnu Rueni (Boşcaiu N., 1965, 1971),

Iris spuria L.: G, Pn-D; U4T3,5R5, Molinietalia, Puccinellietalia: Albina (Grigore Stere, 1971), Liebling (Pop I., 1968)

\section{Orchidaceae}

Dactylorhiza cordigera (Fries) Soo (Orchis cordigera Fries) ssp. cordigera: G, Carp-B; U4.5T2R2, Montio-Cardaminetalia, Scheuchzerio-Caricetalia nigrae: Mt. Mic (Flora XII, Boşcaiu N., 1971a); f. macrobracteata Schur: Groapa Bistrei (Flora XII)

Dactylorhiza incarnata (L.) Soo: G, Eua(M); U4,5T0R4, Calthion, Molinion: Borlova, Coşova, Jdioara, Lugoj (Flora XII)

Epipactis palustris (L.) Crantz: G, Eua; U4.5T3R4.5, Caricetalia davallianae, Eriophorion latifolii, Molinion coeruleae: Armeniş, Borlova (Flora XII), Obreja, Pâr. Hidegu, Poiana Mărului (Boşcaiu N., 1971)

Orchis laxiflora Lam. ssp. elegans (Heuff.) Soo: G, Eua(M); U4T3R0, Molinietalia, Magnocaricion, Eriophorion latifolii: between Ţiglărie-Caransebeş and Racoviţa railwaistations, Pâr. Hidegu, Poiana Mărului, Rusca, V. Bistra (Boşcaiu N., 1971)

\section{Juncaceae}

Juncus alpinoarticulatus Chaix: H, Cp; U4T2R2, Caricion canescenti-fusci, Eriophorion latifolii: Mt. Ţarcu (Boşcaiu N., 1971, 1071a)

Juncus articulatus L. (J. lampocarpus Ehrh.): H, Cp; U5T2R0, Agropyro-Rumicion, Calthion palustris, Nanocyperion flavescentis: Armeniş (Boşcaiu N., 1971,!), Borlova (Boşcaiu N., 1971), Bucoşniţa (!), Lugoj (Boşcaiu N., 1966), Petroşniţa (!), Pâr. Hidegu, Poiana Mărului, Râul Lung, Rusca (Boşcaiu N., 1971), Trei Ape (!), Tapia (Boşcaiu N., 1966), Teregova (!), Trei Ape (!), V. Bistra, V. Sebeşului, Var, Zerveşti (Boşcaiu N., 1971)

Juncus atratus Krocker: H, Eua-C; U4T3R4, Agrostion stoloniferae: Caransebeş-Gara Ţiglărie (Boşcaiu N., 1965), Jebel, Mţii Semenic (Flora XI), Lugoj (Boşcaiu N., 1966)

Juncus bufonius L.: Th, Cosm; U4.5T0R3, Bidentetea tripartiti, Nanocyperetalia, Plantaginetalia majoris: Armeniş (Boşcaiu N., 1971,!), Borlova (Boşcaiu N., 1971), Bucoşniţa (!), Liebling (Neacşu A. et al., 2008), Poiana Mărului, Râul Alb, Rusca, V. Bistra, V. Bistra Mărului, V. Sebeşului (Boșcaiu N., 1971)

Juncus compressus Jacq.: G, Eua; U4T3R4, Agropyro-Rumicion, Agrostion stoloniferae, Nanocyperion flavescentis, Plantaginetea majoris, Puccinellio-Salicornietea: Caransebeş (!), Liebling (Flora XI), Trei Ape (!)

Juncus conglomeratus L.: H, Cp; U4.5T3R3, Calthion palustris, Molinietalia, Molinion coeruleae, Scheuchzerio-Caricetalia nigrae: Armeniş (Boşcaiu N., 1971,!), Caransebeş (!), Lugoj (Boşcaiu N., 1966), Pâr. Hidegu, Poiana Mărului, Râul Alb, Rusca, V. Bistra, V. Bistra Mărului, Var, Zerveşti (Boşcaiu N., 1971) 
Juncus effusus L.: H, Cosm; U4.5T3R3, Alnetea glutinosae, Bidentetea tripartiti, Calthion palustris, Molinietalia, Plantaginetea majoris: Armeniş (Boşcaiu N., 1971,!), Borlova (Boşcaiu N., 1971), Caransebeş, Gărâna, Giulvăz (!), Liebling (Neacşu A. et al., 2008), Lugoj (!), Pâr. Hidegu, Poiana Mărului, Râul Alb, Rusca, Sadova Veche (Boşcaiu N., 1971), Tapia (Boşcaiu N., 1966), Teregova (!), Trei Ape (!), V. Bistra, V. Bistra Mărului, V. Sebeşului, Var, Zerveşti (Boşcaiu N., 1971)

Juncus filiformis L.: H (G), Cp (arct-alp); U4.5T2.5R2.5, Caricion canescenti-nigrae: Mţii. Semenic (Flora XI); var. transsilvanicus (Schur) A. et G.: Mt. Mic (Boşcaiu N., 1971a), Mt. Ţarcu (Boşcaiu N., 1971)

Juncus inflexus L.: H, Eua; U4T3.5R4, Agropyro-Rumicion: Armeniş (Boşcaiu N., 1971,!), Caransebeș (!), la Gara Ţiglărie (Boşcaiu N., 1965), Lugoj (Boşcaiu N., 1966,!), Rusca, (Boşcaiu N., 1971), between Zerveşti and Turnu Rueni (Boşcaiu N., 1965, 1971) Juncus thomassii Ten.: H(G), Cp; U4T2,5R3, Molinietalia: Groapa Bistrei (Boşcaiu N., 1971)

\section{Cyperaceae}

Bolboschoenus maritimus (L.) Palla (Scirpus maritimus L.) ssp. maritimus: G (Hh), Cosm; U6T0R4.5, Bolboschoenion maritimi: Diniaş (Stere G., 1971a), Giulvăz (Stere G., 1971a,!), Ivanda (Stere G., 1971a), Liebling (Neacşu A. et al., 2008), Lugoj beside Complexul I (Boşcaiu N., 1966), Peciu Nou (Stere G., 1971a,!), Rudna (Stere G., 1971a), Tapia on Pâr. Ştiuca (Boşcaiu N., 1966)

Carex acuta L. (C. gracilis Curtis): G (Hh), Cp; U5T3R0, Caricion gracilis, Magnocaricion, Alno-Padion: Peciu Nou (Stere G., 1971a,!), Rudna (Stere G., 1971a)

Carex acutiformis Ehrh.: Hh, Eua; U6T3R4, Magnocaricion, Caricion gracilis: Diniaş, Rudna, Uliuc (Stere G., 1971a), Zlagna (Boşcaiu N., 1965)

Carex curta Good. (C. canescens auct. non L.): H, Cp (bor); U5T0R2, Caricion canescentinigrae: Borlova, Izvorul Sebeşului (Boşcaiu N., 1971), Mt. Mic (Boşcaiu N., 1971a), Mt. Ţarcu (Flora XI, Boşcaiu N., 1971, 1971a), Mţii Semenic (Flora XI); f. fallax F. Kurtz.: Mt. Semenic (Flora XI)

Carex distans L.: H, Eua (sAtl-sM); U4T3R4, Agrostion stoloniferae, Eriophorion latifolii, Molinion coeruleae: Liebling (Neacşu A. et al., 2008), Pâr. Hidegu, Rusca, Turnu Ruieni, Zerveşti (Boşcaiu N., 1971)

Carex echinata Murray (C. stelllulata Good.): H, Cp; U5T2R1, Calthion palustris, Caricion canescenti-nigrae, Magnocaricion elatae: Mt. Mic, Mt. Ţarcu (Boşcaiu N., 1971a), Mţii Semenic (Flora XI), Poiana Mărului, V. Bistra, V. Bistra Mărului, V. Sebeşului (Boşcaiu N., 1971); var. gypos (Schk.) Koch: Groapa Bistrei (Flora XI, Boşcaiu N., 1971)

Carex flava L.: H, Cp; U4.5T3R0, Calthion palustris, Caricetalia davallianae, Eriophorion latifolii, Tofieldietalia: Borlova (Boşcaiu N., 1971), Caransebeş-Gara Ţiglărie (Boşcaiu N., 1965), Groapa Bistrei (Boşcaiu N., 1971), Poiana Mărului, V. Bistra, (Boşcaiu N., 1971), between Zerveşti and Turnu Rueni (Boşcaiu N., 1965, 1971)

Carex lasiocarpa Ehrh.: Hh, Cp; U5T2,5R2,5, Caricion lasiocarpae: Mţii Semenic (Flora XI)

Carex nigra (L.) Reichard (C. fusca All.) ssp. dacica (Heuffel.) Soo (C. dacica Heuffel, Carex bigelowii Torrey et Schwein.): G, Carp-B; U0T2R2,5; Caricion canescenti-nigrae: Mt. Ţarcu (Flora XI, Boşcaiu N., 1971, 1971a)

Carex ovalis Good. (C. leporina auct. non L.): H, Cp; U4T2.5R3, Caricion canescenti-nigrae, Molinietalia, Nardetalia: Armeniş (Boşcaiu N., 1971,!), Caransebeş (!), Gara Ţiglărie (Boşcaiu N., 1965), Mt. Mic, Obreja, Pâr. Hidegu, Poiana Mărului, Poiana Nedeii, Râul Lung, Rusca (Boşcaiu N., 1971), Trei Ape (!), V. Bistra, V. Bistra Mărului, Zerveşti (Boşcaiu N., 1971) 
Carex pallescens L.: H, Cp; U3.5T3R3, Molinio-Arrhenatheretea, Nardetalia: Armeniş (Boşcaiu N., 1971,!), Borlova (Boşcaiu N., 1971), Caransebeş-Gara Ţiglărie (Boşcaiu N., 1965), Groapa Bistrei, Ilova (Boşcaiu N., 1971), Mt. Mic (Boşcaiu N., 1971a), Obreja, Pâr. Hidegu, Poiana Mărului, Rusca, Sadova Veche, V. Bistra, V. Bistra Mărului (Boşcaiu N., 1971), between Zervești and Turnu Rueni (Boşcaiu N., 1965,1971)

Carex pauciflora Lightf.: G, Cp (bor); U5T2.5R1, Oxycocco-Sphagnetea, Sphagnion fusci: Mt. Ţarcu (Flora XI, Boşcaiu N., 1971)

Carex pseudocyperus L.: H (Hh), Cp; U6T3R3.5, Alnetea glutinosae, Caricion rostratae, Magnocaricion elatae: Lugoj, the lake in Str. Bocşei (Boşcaiu N., 1966), Pădureni, V. Timişului Mort (Flora XI, Soran V., 1956)

Carex remota L.: H, Cp; U4.5T3R3, Alno-Padion, Fagetalia silvaticae: between Mt. Mic and Mt. Ţarcu (Flora XI, Boşcaiu N., 1971), Rusca (Boşcaiu N., 1971)

Carex riparia L: Hh, Eua; U5T4R4, Magnocaricion, Caricion gracilis: Liebling (Neacşu A. et al., 2008)

Carex rostrata Stokes ssp. rostrata: $\mathrm{H}(\mathrm{Hh}), \mathrm{Cp}$; U5T2R0, Caricion rostratae, Magnocaricion elatae: Mţii Semenic (Flora XI), Trei Ape (!)

Carex vesicaria L.: Hh, Cp; U6T3R4, Caricion gracilis, Magnocaricion elatae: Caransebeş (XI) Carex vulpina L.: H, Eua; U4T3R4, Agropyro-Rumicion, Caricion gracilis, Magnocaricion elatae, Phragmition australis: Armeniş (Boşcaiu N., 1971,!), Bazoş (Stere G., 1971a,!), Caransebeş (!), Foeni (Stere G., 1971a), Liebling (Neacşu A. et al., 2008), Lugoj (Boşcaiu N., 1966,!), Rusca, Sadova Veche, V. Bistra, Var, Zerveşti (Boşcaiu N., 1971)

Carex $x$ tetrastachys Trauntst. (curta x echinata): Mţii Semenic (Flora XI)

Cyperus flavescens Jacq. (Pycreus flavescens (L.) Reichenb.): Th, Cosm; U4.5T0R4, Nanocyperion flavescentis: Armeniş, Borlova (Boşcaiu N., 1971), Caransebeş (!), Cruceni (Stere G., 1971a), Pădureni (Flora XI), Rudna, Şag, (Stere G., 1971a), Turnu Ruieni (Boşcaiu N., 1971), Urseni (Stere G., 1971a), V. Bistra, Zerveşti (Boşcaiu N., 1971)

Cyperus fuscus L.: Th, Eua; U6T3R4, Nanocyperion flavescentis: Bucoşniţa (!), Liebling Liebling (Flora XI, Neacşu A. et al., 2008), Pădureni on Timiş (Flora XI)

Cyperus glaber L. (Chlorocyperus glaber (L.) Palla): Th, Eua (M); U5T3R4,5, Nanocyperion: Liebling, Şipet (Flora XI)

Cyperus glomeratus L. (Chlorocyperus glomeratus (L.) Palla): Hh, Eua (M); U5T3R4, Nanocyperion: Pădureni onTimiş (Flora XI)

Eleocharis acicularis (L.) Roemer et Schultes: H (Hh), Cp; U5.5T0R0, Nanocyperion flavescentis: Albina, Dragşna (Stere G., 1971a), Lugoj beside Complexul I and V. Cinca beside Boldur (Boşcaiu N., 1966), V. Bistra beside Oțelul Roşu (Boşcaiu N., 1971), Uliuc, Urseni (Stere G., 1971a)

Eleocharis carniolica Koch: Th, Alp-Carp-B; U5T0R5, Nanocyperion: Caransebeş to Turnu Ruieni (Boşcaiu N., 1971, Flora XI), Obreja, V. Bistra (Boşcaiu N., 1971)

Eleocharis ovata (Roth) Roem. et Schult.: Th, Cp; U4,5T4R0, Nanocyperion: Lugoj (FRE), Obreja (Boşcaiu N., 1971)

Eleocharis palustris (L.) Roemer et Schultes: G (Hh), Cosm; U5T0R4, Molinietalia, Nanocyperetalia, Phragmitetea: Armeniş (Boşcaiu N., 1971,!), Borlova (Boşcaiu N., 1971), Caransebeş (!), Giulvăz (Stere G., 1971a,!), Liebling in Balta Mare and Balta Mică (Soran V., 1956), Lugoj (Boşcaiu N., 1966), Peciu Nou (Stere G., 1971a,!), Pâr. Hidegu, Poiana Mărului, Rusca (Boşcaiu N., 1971), Trei Ape (!), Turnu Ruieni (Boşcaiu N., 1971), Urseni (Stere G., 1971a), V. Bistra, Var, Zerverşti (Boşcaiu N., 1971), 
Eriophorum latifolium Hoppe: H, Cp; U5T0R4.5, Caricion davallianae, Eriophorion latifolii, Scheuchzerio-Caricetalia nigrae, Tofieldietalia: Mt. Mic (Boşcaiu N., 1971), Mt. Țarcu (Boşcaiu N., 1971a), Poiana Mărului, Râul Lung, Rusca, Sadova Nouă, V. Bistra Mărului, V. Sebeşului (Boşcaiu N., 1971),

Eriophorum vaginatum L.: H, Cp; U4.5T0R1.5, Sphagnion fusci: Izvorul Râului Lung, Mt. Țarcu (Boşcaiu N., 1971)

Schoenoplectus lacustris (L.) Palla (Scirpus lacustris L.): G (Hh), Cosm; U6T3R4, Phragmition australis: Liebling (Neacşu A. et al., 2008) in Balta Mare (Soran V., 1956), Lugoj (Boşcaiu N., 1966,!), Pădureni on Timişul mort (Soran V., 1956), Peciu Nou, Uliuc (Stere G., 1971a)

Schoenoplectus tabernaemontanus: Ivanda, Rudna, Uliuc, Urseni (Stere G., 1971a)

Scirpus supinus L. (Isolepis supina (L.) R. Br.): Th (Hh), Cosm; U4,5T3R0, Nanocyperion: Giroc (Stere G., 1971a), Jebel (Flora XI, Soran V., 1956), Liebling (Soran V., 1956), Peciu Nou (Stere G., 1971a)

Scirpus sylvaticus L.: G, Cp; U4.5T3R0, Alno-Padion, Calthion palustris, Molinietalia, Phragmitetea: Armeniş (Boşcaiu N., 1971,!), Borlova (Boşcaiu N., 1971), Caransebeş (!) at Gara Țiglărie (Boşcaiu N., 1965), Gărâna (!), Lugoj (Boşcaiu N., 1966,!), Pâr. Hidegu, Poiana Mărului, Râul Alb, Râul Lung, Rusca (Boşcaiu N., 1971), Trei Ape (!), Turnu Ruieni, V. Bistra, V. Bistra Mărului, V. Sebeşului (Boşcaiu N., 1971),

\section{Poaceae \\ Agrostis canina L. ssp. canina: H, Eua; U4T3R3, Caricion canescenti-nigrae, Molinio- Arrhenatheretea: Pâr. Hidegu, Poiana Mărului (Boşcaiu N., 1971) Agrostis gigantea Roth ssp. gigantea: H(G), Eua; U4,5T0R4, Phragmitetalia, Calthion: Borlova (Boşcaiu N., 1971)}

Agrostis stolonifera L. ssp. stolonifera: H, Cp; U4T0R0, Agropyro-Rumicion, Agrostion stoloniferae, Alno-Padion, Magnocaricion elatae, Molinion coeruleae: Armeniş, Bolvaşniţa (Boşcaiu N., 1971,!), Borlova (Boşcaiu N., 1971), Bucoşniţa (!), Caransebeş (!) at Gara Ţiglărie (Boşcaiu N., 1965), Gărâna, Giulvăz (!), Glimboca, Oţelul Roşu, (Boşcaiu N., 1971,!), Ilova (Boşcaiu N., 1966), Jebel (Soran V., 1956), Liebling (Neacşu A. et al., 2008, Soran V., 1956), Lugoj (Boşcaiu N., 1966), Obreja, Pâr. Hidegu, Rusca, Sadova Veche, (Boşcaiu N., 1971), Tapia (Boşcaiu N., 1966), Şag, Trei Ape (!), V. Bistra, V. Bistra Mărului, V. Sebeşului, Vârciorova (Boşcaiu N., 1971), between Zerveşti and Turnu Rueni (Boşcaiu N., 1965, 1971), Zlagna (Boşcaiu N., 1965)

Alopecurus aequalis Sobol.: Th-TH, Cp; U5T3R4, Agrostion stoloniferae, Bidentetalia tripartiti, Nanocyperion flavescentis: Liebling (Neacşu A. et al., 2008) in Balta Mare and Balta Mică (Soran V., 1956)

Alopecurus geniculatus L.: Th-TH, Cosm; U5T0R4, Agropyro-Rumicion, Agrostion stoloniferae, Plantaginetea majoris: Mt. Mic (Boşcaiu N., 1971,1971a); f. natans: Lugoj (Boşcaiu N., 1966)

Alopecurus pratensis L. ssp. pratensis: H, Eua; U4T3R0, Agrostion stoloniferae, Calthion palustris, Filipendulo-Petasition, Molinio-Arrhenatheretea: Armeniş, Bolvaşniţa (Boşcaiu N., 1971,!), Borlova (Boşcaiu N., 1971), Caransebeş (!), Gara Ţiglărie (Boşcaiu N., 1965), Giulvăz (!), Ilova (Boşcaiu N., 1971), Liebling (Neacşu A. et al., 2008), Oţelul Roşu (!), Obreja, Pâr. Hidegu, Râul Alb, Râul Lung, Rusca, Sadova Veche, (Boşcaiu N., 1971), V. Bistra, V. Bistra Mărului, V. Sebeşului, Var, Vârciorova, Zlagna (Boşcaiu N., 1971), between Zerveşti and Turnu Rueni (Boşcaiu N., 1965, 1971)

Beckmannia eruciformis (L.) Host: H, Cp; U4,5T3R4, Beckmannion: Ciacova (Flora XII) 
Calamagrostis canescens (Weber) Roth: H, Eua; U5T3R3, Alnion, Phragmitetea: Liebling (Neacşu A. et al., 2008)

Calamagrostis pseudophragmites (Haller fil.) Koeler: H, Eua-C; U5T3R5, Salicion eleagni: Armeniş, V. Bistra (Boşcaiu N., 1971)

Catabrosa aquatica (L.) Beauv.: H, Cp; UTR, Bidentetea, Glycerio-Sparganion: Armeniş, Rusca, Sadova Veche, Turnu Ruieni, V. Bistra, Zerveşti (Boşcaiu N., 1971)

Deschampsia caespitosa (L.) Beauv. ssp. caespitosa: H, Cosm; U4T0R0, BetuloAdenostyletea, Molinietalia, Phragmitetalia: Caransebeş-Gara Ţiglărie (Boşcaiu N., 1965), Gărâna (!), Trei Ape (!), between Zerveşti and Turnu Rueni (Boşcaiu N., 1965), Mt. Mic, Mt. Ţarcu, V. Bistra Mărului (Boşcaiu N., 1971, 1971a)

Glyceria maxima (Hartman) Holmberg (G. aquatica (L.) Wahlb.): H (Hh), Cp; U5T3R4, Phragmition australis: Bazoş, Chizătău (Stere G., 1971a,!), Liebling (Neacşu A. et al., 2008), between Lugoj and Tapia on Pâr. Ştiuca (Boşcaiu N., 1966), Peciu Nou, Urseni (Stere G., 1971a)

Glyceria fluitans (L.) R.Br. H(Hh), Eua; U5T3R0, Glycerio-Sparganion : Liebling in Balta Mare and Balta Mică (Soran V., 1956), Urseni (Stere G., 1971a)

Glyceria nemoralis (Uechtr.) Uechtr. et Koernicke: H, Ec; U5T3R3, Cardamini-Montion: Mt. Mic (Boşcaiu N., 1971a), Poiana Mărului (Boşcaiu N., 1971)

Glyceria notata Chevall. (G. plicata (Fries) Fries): H (Hh), Cp; U6T3R4.5, GlycerioSparganion: Armneiş (Boşcaiu N., 1971,!), Borlova (Boşcaiu N., 1971), Moşniţa Veche, Peciu Nou (Stere G., 1971a), Trei Ape (!), Turnu Ruieni, V. Bistra, V. Bistra Mărului (Boşcaiu N., 1971)

Crypsis alopecuroides (Piller et Mitterp.) Schrad. (Heleochloa alopecuroides (Piller et Mitterp.) Host: Th, Eua; U4T4R4,5,Cypero-Spergularion : Jebel, Liebling (Soran V., 1956)

Leersia oryzoides (L.) Swartz: G (Hh), Cp; U6T3R0, Bidentetea tripartiti, GlycerioSparganion: Glimboca, Obreja, V. Bistra (Boşcaiu N., 1971); f. patens Wiesb.: Lugoj (Flora XII), V. Ştiuca beside Tapia (Boşcaiu N., 1966)

Molinia coerulea (L.) Moench ssp. coerulea: H, Eua; U4T3R0, Molinion coeruleae: Borlova (Boşcaiu N., 1971), Caransebeş-Gara Ţiglărie (Boşcaiu N., 1965), Faţa Sadovei (Boşcaiu N., 1965, 1971), Lugoj at the brick yard Bartoş (Boşcaiu N., 1966, Flora XI), Pâr. Hidegu, Poiana Mărului, Rusca, V. Bistra, (Boşcaiu N., 1971), between Zerveşti and Turnu Rueni (Boşcaiu N., 1965, 1971), Zlagna (Boşcaiu N., 1965, 1971)

Phalaris arundinacea L. (Typhoides arundinacea (L.) Moench): Hh, Cp; U5T3R0, Agrostion stoloniferae, Caricion gracilis: Bazoş (!), Chizătău, Giroc (Stere G., 1971a), Liebling (Neacşu A. et al., 2008), Peciu Nou (Stere G., 1971a,!), Rusca, Turnu Ruieni (Boşcaiu N., 1971), Urseni (Stere G., 1971a), V. Bistra (Boşcaiu N., 1971), V. Ştiuca beside Tapia (Boşcaiu N., 1966)

Phragmites australis (Cav.) Steudel ssp. australis: G (Hh), Cosm; U6T0R4, Phragmition australis: Bazoş, Belinţ, Bucoşniţa (!), Caransebeş (!) at Gara Ţiglărie (Boşcaiu N., 1965), Chizătău, Coşteiu (!), Cruceni, Diniaş, Foeni, Giroc (Stere G., 1971a), Giulvăz (!), Ivanda (Stere G., 1971a), Liebling (Neacşu A. et al., 2008), Lugoj (Boşcaiu N., 1966,!), Lugojel (!), Peciu Nou, Rudna (Stere G., 1971a), Sacu (!), Şag (Stere G., 1971a,!), Uliuc, Urseni (Stere G., 1971a); f. subuniflora DC.: V. Timişului (jud. Timiş) (Flora XII)

Poa palustris L.: H, Cp; U5T3R4, Alnetalia glutinosae, Calthion palustris, Magnocaricion elatae, Phragmition australis: Armeniş (Boşcaiu N., 1971), Giroc (Al. Buia, 1942), Liebling 
(Neacşu A. et al., 2008), Lugoj, Tapia (Boşcaiu N., 1966), V. Bistra (Boşcaiu N., 1971); var. effusa Rchb.: Giroc (Al. Buia, 1942); var. fertilis Rchb.: Giroc (Al. Buia, 1942)

\section{Sparganiaceae}

Sparganium erectum L. (S. ramosum Hudson) ssp. erectum: G (Hh), Eua; U5.5T3.5R0, Glycerio-Sparganion, Phragmition australis: Liebling in the fen Tofaia and Balta Mare (Soran V., 1956), Lugoj (Boşcaiu N., 1966), Pădureni on Timişul Mort and in puddles (Soran V., 1956), Urseni (Soran V., 1956); ssp. neglectum (Beeby) K. Richter: G, Hh, Ec: Armeniş (Boşcaiu N., 1971), Obreja, Sadova Veche (Boşcaiu N.,1971), Urseni (Stere G., 1971a), V. Bistra, var. (Boşcaiu N.,1971); ssp. oocarpum (Celak) Domin: Urseni (Grigore. Stere, 1971)

\section{Typhaceae}

Typha angustifolia L.: G (Hh), Cp; U6T4R0, Phragmition australis: Glimboca (Boşcaiu N.,1971), Liebling (Neacşu A. et al., 2008), in fen Tofaia (Soran V., 1956), Lugoj (Boşcaiu N., 1966), Pădureni on Timişul Mort and in puddles (Soran V., 1956), Urseni (Soran V., 1956, Stere G., 1971a)

Typha latifolia L.: G (Hh), Cosm; U6T3.5R0, Phragmition australis: Belinţ, Bucoşniţa (!), Caransebeş, Giulvăz (!), Glimboca (Boşcaiu N.,1971), Liebling (Neacşu A. et al., 2008) in fen Tofaia (Soran V., 1956), Lugoj (Boşcaiu N., 1966), Pădureni on Timişul Mort and in puddles (Soran V., 1956), Urseni (Soran V., 1956, Stere G., 1971a), Trei Ape (!)

Typha shuttleworthii Koch et Sonder: G (Hh), E (mont); U6T3R0, Phragmition australis: Izvorul Bistrei Mărului, Iyvorul Sebeşului (Boşcaiu N.,1971), Moşniţa (Flora XI), Râul Lung, Rusca (Boşcaiu N.,1971)

\section{Araceae}

Acorus calamus L. G (Hh), Adv; U6T3,5R4, Phragmitetalia: Lugoj (Flora XII)

\section{Lemnaceae}

Lemna gibba L.: Hh, Cosm; U6T3,5R4, Lemnion minoris: Liebling (Flora XII) in Balta Mare and Balta Mică (Soran V., 1956)

Lemna minor L.: Hh, Cosm; U6T0R0, Lemnion minoris: Albina (Stere G., 1971a), Bazoş (Stere G., 1971a,!), Belinţ, Caransebeş, Criciova (!), Chizătău (Stere G., 1971a,!), Cruceni, Foeni, Giroc, Giulvăz (!), Ivanda (Stere G., 1971a), Liebling (Neacşu A. et al., 2009) in fen Tofaia and Balta Mare and Balta Mică (Soran V., 1956), Lugoj (Boşcaiu N., 1966,!), Lugojel (!), Pădureni on Timişul Mort and in puddles (Soran V., 1956), Peciu Nou, Rudna (Stere G., 1971a), Şag (Stere G., 1971a,!), Tapia (Boşcaiu N., 1966), Uliuc, Urseni (Stere G., 1971a)

Lemna trisulca L.: Hh, Cosm; U6T0R4, Lemnion minoris: Giroc, Foeni (Stere G., 1971a), Lugoj, the lake in Str. Bocşei and at the brick yard Bartoş (Boşcaiu N., 1966), Pădureni on Timişul Mort (Soran V., 1956), Uliuc, Urseni (Stere G., 1971a)

Spirodela polyrhiza (L.) Schleiden: Hh, Cosm; U6T3.5R0, Lemnion minoris: Bazoş, Giulvăz, (Stere G., 1971a), Liebling (Neacşu A. et al., 2008) in fen Tofaia and Balta Mare (Soran V., 1956), Lugoj in Pâr. Ştiuca (Boşcaiu N., 1966), Pădureni on Timişul Mort and in puddles (Soran V., 1956), Peciu Nou (!), Şag, Uliuc, Urseni (Stere G., 1971a)

Wolffia arrhiza (L.) Horkel: Hh, Cosm; U6T0R4, Lemnion minoris: lunca Timişului la Albina, Giroc (Stere G., 1971a), Liebling (Flora XII), Liebling in puddle Balta Mare (Soran V., 1956), Lugoj (Flora XII), lake in Str. Bocşei (Boşcaiu N., 1966), Pădureni on Timişul Mort (Soran V., 1956), Uliuc, Urseni (Stere G., 1971a) 


\section{Vegetation}

Agrostidetum stoloniferae (Ujvárosi 1941) Burduja et al. 1956: Caransebeş-Gara Ţiglărie (Boşcaiu N., 1965), Liebling (Neacşu A. et al., 2009)

Bolboschoenetum maritimi (Soo 1957) Eggler 1933: Diniaş (Stere G., 1971a), Giulvăz (Stere G., 1971a,!), Ivanda (Stere G., 1971a), Lugoj (Boşcaiu N., 1966), Peciu Nou, Rudna (Stere G., 1971a)

Calthaetum laetae Krajina 1933: Mt. Mic, Mt. Țarcu, V. Bistra Mărului (Boşcaiu N., 1971a)

Cardaminetum opicii Szafer, Pawl. Kulcz. 1923: Groapa Bistrei, Mt. Mic (Boşcaiu N., 1971a)

Caricetum acutiformis Eggler 1933 (Caricetum acutiformis-ripariae Soo (1938) 1947):

Caransebeș-Gara Ţiglărie (Boşcaiu N., 1965), Diniaş, Rudna, Uliuc (Stere G., 1971a)

Caricetum gracilis (Graebn. et Hueck 1931) Almquist 1929: Peciu Nou (Stere G., 1971a,!), Rudna (Stere G., 1971a)

Caricetum rostratae Rubel 1912: Trei Ape (!)

Caricetum vulpinae (Nowinski 1928) Soo 1927: Caransebeş (!), Bazoş, Foeni (Stere G., 1971a)

Ceratophyllo-Hydrocharitetum Pop I., 1962: Chizătău (Stere G., 1971a), Lugoj (Boşcaiu N., 1966)

Ceratophylletum demersi Hild 1956: Liebling (Neacşu A. et al., 2009)

Chrysosplenio-Cardaminetum (Tx. 1937) Maas 1959: V. Bistra Mărului, V. Sebeşului (Boşcaiu N., 1971a)

Cyperetum flavescentis W. Koch 1926: Caransebeş (!), Cruceni, Rudna, Şag (Stere G., 1971a,!), Urseni (Stere G., 1971a)

Deschampsietum caespitosae Hayek ex Horvatic 1930: Caransebeş-Gara Ţiglărie (Boşcaiu N., 1965)

Eleocharidetum acicularis (W. Koch 1926) R. Tx. 1937: Albina, Dragşna (Stere G., 1971a), Boldur, Lugoj (Boşcaiu N., 1966), Uliuc, Urseni (Stere G., 1971a)

Eleocharitetum palustris (Schennikow 1919) Ubrizsy 1948 (Alismato-Eleocharetum Mathe et Kovacs 1967): Caransebeș (!), Cruceni, Diniaş, Giulvăz, Peciu Nou (Stere G., 1971a,!), Urseni (Stere G., 1971a)

Filipendulo-Geranietum palustris W. Koch 1926 subas. filipenduletosum: Trei Ape (!), Gărâna (!)

Galio palustris-Caricetum ripariae Balatova-Tulačkova el al. 1993 (Caricetum ripariae soo 1928; Knapp et Stoffer 1962): Liebling (Neacşu A. et al., 2009)

Glycerietum fluitantis Eggler 1933 (Sparganio-Glycerietum fluitantis Br.-Bl. 1925, GlycerioSparganietum neglecti Koch 1926): Caransebeş-Gara Ţiglărie (Boşcaiu N., 1965) Pădureni on Timişul Mort (Soran V., 1956), Urseni (Soran V., 1956, Stere G., 1971a)

Glycerietum maximae (Nowinski 1930) Hueck 1931): Bazoş, Chizătău (Stere G., 1971a), Liebling (Neacşu A. et al., 2009), Lugoj (Boşcaiu N., 1966), Peciu Nou, Urseni (Stere G., 1971a)

Glycerietum plicatae Kulczynski 1928 (Oberd. 1954): Moşniţa Veche, Peciu Nou (Stere G., 1971a)

Helianthetum decapetali Morariu 1967: Bucoşniţa (!), Petroşniţa (!)

Hydrochari-Stratiotetum (Langend. 1935) Westhoff 1942: Uliuc (Stere G., 1971a)

Iridetum pseudacori Eggler 1933: Liebling (Neacşu A. et al., 2009)

Juncetum effusi Soo (1931) 1949: Teregova (!) 
Junco (inflexi)-Menthetum longifoliae Lohm. 1953.: Trei Ape (!)

Lemnetum minoris Oberd. 1957 ex Muller et Gors 1960: Albina (Stere G., 1971a), Bazoş (Stere G., 1971a,!), Belinţ, Caransebeş, Criciova (!), Chizătău (Stere G., 1971a,!), Cruceni, Foeni, Giroc, Giulvăz (!), Ivanda (Stere G., 1971a), Liebling (Neacşu A. et al., 2009) Lugoj (Boşcaiu N., 1966,!), Peciu Nou, Rudna (Stere G., 1971a), Şag (Stere G., 1971a,!), Tapia (Boşcaiu N., 1966), Uliuc, Urseni (Stere G., 1971a)

Lemno-Utricularietum Soo (1928) 1947: Belinţ, Chizătău, Foeni, Giroc (Stere G., 1971a), Lugoj (Boşcaiu N., 1966), Rudna, Uliuc, Urseni (Stere G., 1971a); facies cu Spirodela polyrrhiza: Liebling in fen Tofaia and in Balta Mare (Soran V., 1956), Pădureni in Timişul Mort (Soran V., 1956); facies cu Salvinia natans: Pădureni in Timişul Mort (Soran V., 1956); facies cu Wolfia arrhiza: Pădureni in Timişul Mort (Soran V., 1956)

Lindernio-Isolepetum Morariu 1943 (Eleochatis acicularis-Schoenoplectus supinus Soo 1951 p.p., Eleocharetum ovatae Hayek 1923 p.p.): Cerna, Folia (Soran V., 1956) Giroc (Stere G., 1971a), Jebel, Lebling (Soran V., 1956), Peciu Nou, Şag (Stere G., 1971a)

Lythro-Calamagrostetum epigeii Pop I. 1968: Lugoj (Boşcaiu N., 1966)

Myriophyllo-Potametum Soo 1934: Liebling (Neacşu A. et al., 2009) in Timiş and in Balta Mare and Balta Mică (Soran V., 1956), Lugoj (Boşcaiu N., 1966), Moşniţa Veche, Rudna (Stere G., 1971a), between Şag and Urseni (Soran V., 1956), Urseni (Stere G., 1971a) cu subas. marsileetosum Soo 1957 şi myriophylletosum spicati Soo 1957

Nupharetum albo-luteae Nowinski 1928 (Potameto-Nupharetum T. Müller et Görs. 1960): Lugoj, Tapia (Boşcaiu N., 1966)

Oenantho aquaticae-Rorippetum amphibiae (Soo 1927) Lohm. 1950: în lunca Timişului la Rudna, Uliuc, Urseni (Stere G., 1971a)

Parvipotamo-Zannichellietum W. Koch 1926: Boldur in V. Cinca (Boşcaiu N., 1966), Liebling in Balta Mare and Balta Mică (Soran V., 1956), Rudna (Stere G., 1971a)

Petasitetum hybridi (Dostal 1933) Soo 1940: Trei Ape (!)

Peucedano rocheliani-Molinietum coeruleae Boşcaiu, 1965: Caransebeş-Gara Țiglărie (Boşcaiu N., 1965), between Zerveşti and Turnu Rueni (Boşcaiu N., 1965), Zlagna (Boşcaiu N., 1965)

Phalaridetum arundinaceae (Horvatiè 1931) Libbert 1931: Bazoş (!), Chizătău, Giroc (Stere G., 1971a), Liebling (Neacşu A. et al., 2009), Peciu Nou (Stere G., 1971a,!), Tapia (Boşcaiu N., 1966), Urseni (Stere G., 1971a)

Philonotido-Saxifragetum stellaris Horv. 1949: Groapa Bistrei, Mt. Mic (Boşcaiu N., 1971a) Phragmitetum vulgaris Soo 1927 (Scirpo-Phragmitetum W. Koch 1926): Bazoş, Belinţ, Bucoşniţa (!), Caransebeş (!) at Gara Țiglărie (Boşcaiu N., 1965), Chizătău, Coşteiu (!), Cruceni, Diniaş, Foeni, Giroc (Stere G., 1971a), Giulvăz (!), Ivanda (Stere G., 1971a), Liebling (Neacşu A. et al., 2008), Lugoj (Boşcaiu N., 1966,!), Lugojel (!), Peciu Nou, Rudna (Stere G., 1971a), Sacu (!), Şag (Stere G., 1971a,!), Uliuc, Urseni (Stere G., 1971a)

Poëtum pratensis Rãv., Cãzãc. et Turenschi ex Răv. et Mititelu 1958 : Liebling (Neacşu A. et al., 2009)

Polygonetum cuspidati Tüxen et Raabe 1950: Armeniş (!), Bucoşniţa (!), Petroşniţa (!), Sadova Veche (!)

Polygono hydropiperi-Bidentetum tripartiti Lohm. 1950 (Bidentetum tripartiti W. Koch 1926, Polygonetum hydropiperi Passarge 1965): Bucoşniţa (!)

Polygono - Potametum natantis Soó 1964: Chizătău (Stere G., 1971a,!), Cruceni (Stere G., 1971a), Liebling (Neacşu A. et al., 2009), Peciu Nou, Rudna, Şag (Stere G., 1971a); subas. potametosum natantis Soo 1964: Bazoş (!), Lugoj (Boşcaiu N., 1966); subas. polygonetosum amphibii Soo 1924: Boldur, Lugoj (Boşcaiu N., 1966) 
Potamo-Ceratophylletum Pop I., 1962: Cruceni, Diniaş, Foeni, Giroc, Moşniţa Veche, Rudna (Stere G., 1971a)

Pulicario vulgaris-Menthetum pulegii Slavnic 1951: Caransebeş (!), Cruceni, Foeni, Giroc (Stere G., 1971a), Giulvăz (Stere G., 1971a,!), Ivanda (Stere G., 1971a), Lugoj (Boşcaiu N., 1966,!), Rudna (Stere G., 1971a), Şag, Teregova (!), Urseni (Stere G., 1971a)

Ranunculo trichophylli-Callitrichetum cophocarpae (Soo 1927) Pocs 1958: Caransebeş-Gara Ţiglărie (Boşcaiu N., 1965)

Ranunculetum aquaticae Gehu 1961: Dragşna, Foeni (Stere G., 1971a)

Ranunculetum lateriflori Pop I. 1962: Diniaş, Giulvăz (Stere G., 1971a)

Ranunculetum repentis Knap 1946 emend. Oberd. 1957: Belinţ, Caransebeş, Chizătău, Giulvăz, Lugoj, Peciu Nou (!)

Ranunculo repenti-Alopecuretum pratensis Ellmauer et Mucina 1993 (Alopecuretum pratensis Regel 1925, Agrosti-Alopecuretum pratensis Ubrizsi 1955): Caransebeş-Gara Ţiglărie (Boşcaiu N., 1965), Liebling (Neacşu A. et al., 2009)

Rubo - Salicetum cinereae Sonasak 1963 : Liebling (Neacşu A. et al., 2009)

Salicetum albae Issler 1926: Bucoşniţa (!)

Salvinio-Hydrocharitetum (Oberd. 1957) Boşcaiu 1966: Cruceni, Chizătău, Giroc, Ivanda (Stere G., 1971a), Lugoj (Boşcaiu N., 1966), Uliuc, Urseni (Stere G., 1971a)

Salvinio-Spirodeletum Slav. 1956: Bazoş (Stere G., 1971a), Peciu Nou (!), Şag, Uliuc, Urseni (Stere G., 1971a)

Saxifragetum aizoidis Horv. 1935: Dunga Gropii Bistra under Vf. Țarcu (Boşcaiu N., 1971a)

Scirpetum lacustris Chouard 1924 (Schoenoplectetum lacustris Eggler 1933): Liebling (Neacşu A. et al., 2009), Lugoj (Boşcaiu N., 1966), Peciu Nou, Uliuc (Stere G., 1971a)

Scirpetum silvatici Ralski 1931: Gărâna (!), Trei Ape (!)

Scirpetum tabernaemontani (Soo 1949) Pass. 1964: Ivanda, Rudna, Uliuc, Urseni (Stere G., 1971a)

Lemno-Spirodelatum polyrhizae W. Koch 1926 (Spirodeletum polyrrhizae W. Koch 1954): Liebling (Neacşu A. et al., 2009), Lugoj on Pâr. Ştiuca (Boşcaiu N., 1966)

Stellario nemorum-Alnetum glutinosae Lohmeyer 1957 (Aegopodio-Alnetum glutinosae J. Karpati et Jurko 1961): Armeniş (!), Gărâna (!), Teregova (!), Trei Ape (!)

Trapetum natantis (Müller et Görs 1960) V. Karpati 1963: Liebling (Neacşu A. et al., 2009), Lugoj (Boşcaiu N., 1966)

Typhaetum angustifoliae Pignatti 1953: Liebling (Neacşu A. et al., 2009), Lugoj (Boşcaiu N., 1966)

Typhaetum latifoliae G. Lang 1973: Caransebeş (!), Cruceni (Stere G., 1971a), Giulvăz (!), Liebling (Neacşu A. et al., 2009), Lugoj (!), Trei Ape (!), Urseni (Stere G., 1971a)

Wolffietum arrhizae Miyawaki et J. Tx. 1960: Albina, Giroc (Stere G., 1971a), Lugoj, the lake in Str. Bocşei (Boşcaiu N., 1966), Uliuc, Urseni (Stere G., 1971a)

\section{CONCLUSIONS}

In the Timiş River drainage basin, the aquatic and paludal flora and vegetation are quite numerous, as a consequence of the multitude of ponds, lakes and swamps.

Out of the 285 species identified, more than twenty are either rare or protected. Between them there are typical water macrophytes, species prevalent in sites with changing water levels, or temporary dry ares as well as species of swamps and of meadows with changing wetness (e.g. Thelypteris palustris, Marsilea quadrifolia, Nuphar lutea, Myosurus minimus, Ranunculus lateriflorus, Ranunculus lingua, Ranunculus ophioglossifolius, Montia 
minor, Rumex x gayeri, Ludwigia palustris, Apium nodiflorum, Peucedanum rochelianum, Elatine hexandra, Tozzia alpina ssp. carpatica, Cirsium brachycephalum, Taraxacum bessarabicum, Stratiotes aloides, Fritillaria meleagris, Gladiolus imbricatus, Narcissus poëticus ssp. radiiflorus, Wolffia arrhiza).

Beside the larger spread plant coenoses coenenoses, in the area there are found plant communities with a limited distribution in Romania such are Eleocharidetum acicularis, Lindernio-Isolepetum, Peucedano rocheliani-Molinietum coeruleae, Ranunculetum lateriflori and Wolffietum arrhizae.

\section{ACKNOWLEDGEMENTS}

A part of this work resulted from the scientific study of the quality of the Timiş River water, "Study of the quality of Timiş water and registry of polluters source to border" (IV.1.2), a Phare CBS contract, no. 411 - 90964/30.12.2010/07 of "Lucian Blaga" University, Faculty of Sceinces, financed by the European Community.

\section{SELECTED REFERENCES}

1. Arvat N., 1977 - Flora şi vegetaţia dintre râurile Timiş, Pogoniş şi Bârzava, Teză de doctorat, Iaşi. (in Romanian)

2. Borbás V., 1884 - Flora comitatus temesiensis, Timişoara, 53. (in Hungarian)

3. Boşcaiu N., 1942 - Flora luncii Sebeşului de lângă Zerveşti, Natura, 31, 64-66. (in Romanian)

4. Boşcaiu N., 1944 - Contribuţii la flora şi vegetaţia Muntelui Mic din Banat, Primăvara Banatului, Lugoj, 5, 8-13. (in Romanian)

5. Boşcaiu N., 1965 - Cercetări fitocenologice asupra asociaţiei Peucedano - Molinierum coeruleae din Banat şi Țara Haţegului, Contribuții Botanice Cluj, 251-264. (in Romanian)

6. Boşcaiu N., 1966 - Vegetaţia acvatică şi palustră din împrejurimile oraşului Lugoj, Contribuții Botanice Cluj, II, 69-80

7. Boşcaiu N., 1971 - Flora şi vegetaţia Munţilor Ţarcu, Godeanu şi Cernei, Edit. Academiei Române, Bucureşti, 494. (in Romanian)

8. Boşcaiu N., 1971a - Vegetaţia fontinală din Munţii Ţarcu, Godeanu şi Cernei, Studii şi Comunicări Muzeul Brukenthal Sibiu, Ştiințele Naturii, 16, 124-133.

9. Buia A., 1942 - Contribuţii la flora Timişoarei, Buletinul Grădinii Botanice Cluj, XXII, 57-62

10. Bujorean G., 1959 - Montia verna în flora Banatului, Studii şi cercetări de Biologie, Seria ştiințe agricole, Timişoara, VI, 3-4, 91-95.

11. Stere G. 1971 - Flora şi vegetaţia din interfluviul Timiş-Bega, Teză de doctorat, Institutul Agronomic „Ion Ionescu de la Brad”, Iaşi.

12. Stere G., 1971a - Vegetaţia acvatică şi palustră din interfluviul Timiş-Bega, Studii şi cercetări de Biologie, Seria Botanică, 23, 1, 13-46.

13. Heuffel J., 1858 - Enumeratio plantarum in Banatu Temesiensis sponte crescentium et frequentius cultorum, Vindobonae. (in Francais)

14. Neacşu A., Arsene G. -G., Fărcăşescu A., Faur F. and Stroia C., 2008 - Aquatic and paludicolous vegetation from some Banat sites, Lucrări ştiinţifice, Timişoara, 40, 55-60

15. Neacşu A., Arsene G. -G., Fărcăşescu A. and Faur F., 2009 - The vegetation of the lake Liebling (Timiş County), Lucrări ştiinţifice Facultatea de Agronomie, Timişoara, 41, 2, 279-284.

16. Pop A., 1977 - Flora şi vegetaţia din Câmpia joasă a Timişului. Studiu floristic, ecologic şi geobotanic, Teză de doctorat, Universitatea Cluj-Napoca.

17. Pop I., 1968 - Plante noi şi rare din Banat, Studia Universitatis Babeş-Bolyai Cluj, 3-6.

18. Rochel A., 1823 - Plantae Banatus rariores, Pestinii.

19. Săvulescu T. (ed.), 1952-1976 - Flora R. P. R. I- XIII, Edit. Academiei Române, Bucureşti.

20. Soran V. 1954 - Flora de la Liebling şi împrejurimi, Studii şi cercetări de Biologie, Cluj, 5, 1-2.

21. Soran V., 1956 - Câteva asociaţii de plante acvatice şi palustre din Banat, Studii şi cercetări de Biologie, Cluj, 7, 1-4, 107-124

22. Vicol E. C., 1974 - Flora şi vegetaţia Piemontului Lugojului, Teză de doctorat, Universitatea Cluj-Napoca. 